\title{
A high-order Petrov-Galerkin finite element method for the classical Boussinesq wave model
}

\author{
Paulo Avilez-Valente ${ }^{1, *, \dagger}$ and Fernando J. Seabra-Santos ${ }^{2}$ \\ ${ }^{1}$ Faculdade de Engenharia, CEHRA, Universidade do Porto, Rua Dr Roberto Frias, P-4200-465 Porto, Portugal \\ ${ }^{2}$ Departamento de Engenharia Civil, Universidade de Coimbra, Rua Luís Reis Santos, Pólo II da Universidade, \\ P-3030-788 Coimbra, Portugal
}

\begin{abstract}
SUMMARY
A high-order Petrov-Galerkin finite element scheme is presented to solve the one-dimensional depthintegrated classical Boussinesq equations for weakly non-linear and weakly dispersive waves. Finite elements are used both in the space and the time domains. The shape functions are bilinear in spacetime, whereas the weighting functions are linear in space and quadratic in time, with $C^{0}$-continuity. Dispersion correction and a highly selective dissipation mechanism are introduced through additional streamline upwind terms in the weighting functions. An implicit, conditionally stable, one-step predictorcorrector time integration scheme results. The accuracy and stability of the non-linear discrete equations are investigated by means of a local Taylor series expansion. A linear spectral analysis is used for the full characterization of the predictor-corrector inner iterations. Based on the order of the analytical terms of the Boussinesq model and on the order of the numerical discretization, it is concluded that the scheme is fourth-order accurate in terms of phase velocity. The dissipation term is third order only affecting the shortest wavelengths. A numerical convergence analysis showed a second-order convergence rate in terms of both element size and time step. Four numerical experiments are addressed and their results are compared with analytical solutions or experimental data available in the literature: the propagation of a solitary wave, the oscillation of a flat bottom closed basin, the oscillation of a non-flat bottom closed basin, and the propagation of a periodic wave over a submerged bar. Copyright (C) 2008 John Wiley \& Sons, Ltd.
\end{abstract}

Received 22 May 2007; Revised 1 April 2008; Accepted 17 April 2008

KEY WORDS: Boussinesq equations; finite element method; Petrov-Galerkin; dispersive waves; nonlinear waves; stability analysis; accuracy analysis

\footnotetext{
*Correspondence to: Paulo Avilez-Valente, Faculdade de Engenharia, CEHRA, Universidade do Porto, Rua Dr Roberto Frias, P-4200-465 Porto, Portugal.

${ }^{\dagger}$ E-mail: pvalente@fe.up.pt

Contract/grant sponsor: Fundação para a Ciência e a Tecnologia; contract/grant number: POCTI \ECM \41800\2001
} 


\section{P. AVILEZ-VALENTE AND F. J. SEABRA-SANTOS}

\section{INTRODUCTION}

In recent years, an important research effort has been put into the mathematical development of non-linear and dispersive wave models. The original works of Boussinesq [1], Serre [2], and Peregrine [3] were formulated in terms of the free-surface elevation and a horizontal velocity variable, and were limited to non-breaking and weakly non-linear waves, in shallow waters. Since then several extensions have been made to account for wave-breaking dissipation [4-9], deep water propagation [10-15], and full non-linearity $[11,15]$. Nevertheless, these models were limited to $k h<6$ for surface quantities, and $k h<4$ for vertical velocity distributions [16]. In Reference [17] a vertical velocity is retained as an unknown, and for a fifth-order model highly non-linear waves can be simulated for $k h<25$, with accurate velocity profiles for $k h<12$.

Traditionally, high-order finite difference methods have been used to solve these equations, from the Peregrine model $[3,18,19]$ to the most elaborate Serre model [20], and enhanced Boussinesq models [11,21]. A linear and non-linear stability analysis of some of these schemes is provided in Reference [22]. However, difficulties may arise when modelling irregular coastlines and/or rapidly varying depths, which are hard to deal with using structured finite difference grids.

Finite volume techniques have been proposed by several authors, most of them only secondorder accurate, for both kinematic and dynamic dispersive waves [23-25]. Recently, a fourth-order scheme was developed for the Serre equations, which seems to be quite efficient [26,27], although filtering had to be added to ensure stability.

Finite element methods (FEMs) for Boussinesq-type models are becoming more frequently found in the literature, mainly due to its geometrical flexibility. Most of the research work on this subject concerned the classical Boussinesq equations, and second-order (or even first-order) Bubnov-Galerkin (BG) formulations were used [28-30], while in Reference [31] a Taylor-Galerkin FEM scheme was introduced. More recently, a few papers were published on FEM formulations for the enhanced Boussinesq models [6,32-37]. Owing to the presence of additional frequency dispersion terms with third-order space derivatives, auxiliary variables had to be introduced for the usual BG scheme to be applied. In most cases, no accuracy analysis was produced, and at most second-order accuracy might be expected. However, as the lower-order terms associated with the truncation error of the discretized equations may have a similar form than the higher-order terms in the Boussinesq equations, a higher-order scheme is necessary when dealing with dispersive waves [19]. Woo and Liu [38] introduced a Petrov-Galerkin (PG) FEM formulation with $C^{2}$-continuity weighting functions and fourth-order accuracy. However, stability could only be achieved for low Courant numbers $(C r<0.5)$ and a high number of iterations per time step. Moreover, the extension of $C^{2}$-continuity weighting functions for the two-dimensional unstructured grids will not be straightforward. Langtangen and Pedersen [39] presented a high-order FEM formulation where truncation error terms are cancelled through the introduction of correction terms in the discrete equations. However, as those authors pointed out, extension to a model with friction and turbulence terms might be difficult, due to their usage of the velocity potential as a primary variable.

The proposed FEM is based on a PG formulation in both space and time. This discretization is similar to the one introduced in Reference [40] for convective problems. The shape functions are bilinear in space and time with $C^{0}$-continuity, whereas the weighting functions are linear in space and quadratic in time with $C^{0}$-continuity as well. The weak forms of the equations are re-arranged in such a way that only piecewise linear in space functions are present in the final formulation. The coefficients of the two extra upwind terms in the weighting functions are adjusted to eliminate 
A PETROV-GALERKIN FEM FOR THE BOUSSINESQ WAVE MODEL

the second-order dispersion error induced by the convection operator, and thus achieving fourthorder accuracy in the phase velocity, combined with a highly selective third-order dissipation mechanism. The scheme is applied to the one-dimensional classical Boussinesq equations derived by Peregrine [3]. These equations are only valid for weakly non-linear and weakly dispersive water waves. However, the solution of these equations will be the first step towards the application of the presented numerical approach for the solution of the class of Boussinesq equations. Indeed, its $C^{0}$-continuity formulation makes it suitable for extension to two-dimensional problems with unstructured meshes, and extension to the enhanced Boussinesq equations is also feasible, although accuracy will still be limited to the third order.

In Section 2, we present the Boussinesq model and its linear dispersion relation. A short discussion on the bottom gradient terms is included, and some considerations are made on the energy conservation properties of the model. The PG space-time FEM scheme is formulated in Section 3. In Section 4, the stability and accuracy analysis of the proposed method is performed, and the coefficients of the upwind terms are determined. Section 5 is devoted to four case studies: propagation of a solitary wave; oscillation of a flat bottom closed basin; oscillation of a nonflat bottom basin; and wave propagation over a submerged bar. Some stability, convergence, and conservation results are analysed. Comparisons with analytical solutions are provided for the first two cases, while published laboratory results are used for the fourth case study. For the third case, a non-uniform mesh is used. In Section 6 we summarize our conclusions. Finally, an Annex is included for the stability and accuracy analysis for the non-flat bottom case.

\section{GOVERNING EQUATIONS}

The classical Boussinesq equations for weakly non-linear, and weakly dispersive water waves, describe the inviscid and incompressible shallow water flow over uneven bottoms. The model is formulated in terms of the free-surface elevation, $\zeta(x, t)$, and of the depth-averaged horizontal velocity, $\bar{u}(x, t)$, on a coordinate system with horizontal axis, $O X$, in the undisturbed water level, and the vertical axis, $O Z$, pointing upwards. The fluid is vertically confined to

$$
-h(x)<z<\zeta(x, t)
$$

where $h$ is the undisturbed water depth. An average or reference water depth $h_{0}$ can be defined for scaling purposes (see Figure 1). The equations of mass and momentum conservation, originally derived by Peregrine [3], are, respectively

$$
\frac{\partial \zeta}{\partial t}+\frac{\partial}{\partial x}[(h+\zeta) \bar{u}]=0
$$

and

$$
\frac{\partial \bar{u}}{\partial t}+\bar{u} \frac{\partial \bar{u}}{\partial x}+g \frac{\partial \zeta}{\partial x}-\frac{\partial}{\partial t}\left[\frac{h}{2} \frac{\partial^{2}}{\partial x^{2}}(h \bar{u})-\frac{h^{2}}{6} \frac{\partial^{2} \bar{u}}{\partial x^{2}}\right]=0
$$

where $g$ is the gravity acceleration. 


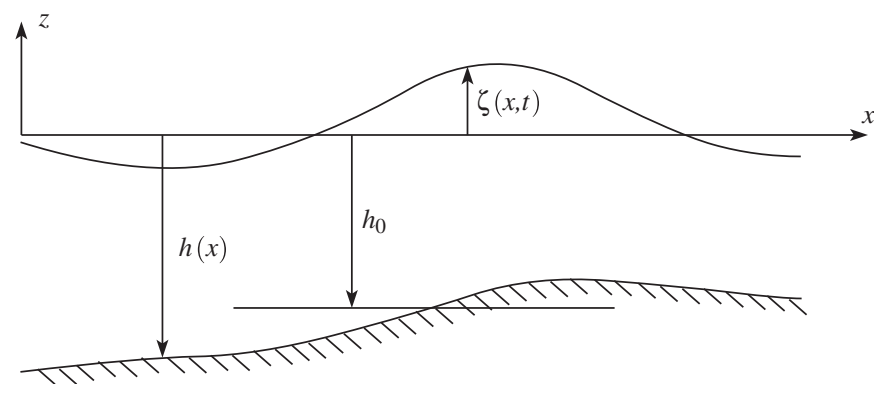

Figure 1. Geometric definition.

\subsection{Dimensionless equations}

Peregrine derived Equations (2) from the Euler equations, assuming the absence of bottom friction and surface stresses. A few scalings were necessary:

$$
\begin{aligned}
& \zeta=a \zeta^{\prime}, \quad u=\varepsilon c u^{\prime}, \quad w=\varepsilon \sigma c w^{\prime}, \quad t=t^{\prime} /(k c) \\
& x=x^{\prime} / k, \quad z=h_{0} z^{\prime}, \quad h=h_{0} h^{\prime}, \quad p=\rho g h_{0} p^{\prime}
\end{aligned}
$$

Herein $a$ is the wave amplitude; $w$ is the vertical velocity; $p$ is the pressure; $\rho$ is the fluid density; $k=2 \pi / \lambda$ is the wave number, with $\lambda$ being the wavelength; and $c=\omega / k$ is the wave velocity, with $\omega$ being the wave angular frequency. The primes indicate dimensionless variables that are assumed to be of $\mathcal{O}(1)$. The small parameters $\varepsilon$ and $\sigma$ are

$$
\varepsilon=a / h_{0} \quad \text { and } \quad \sigma=k h_{0}
$$

which stand, respectively, for a non-linearity parameter and a dispersion parameter. It should be noticed that these scalings impose a mild bottom slope condition:

$$
\frac{\mathrm{d} h}{\mathrm{~d} x}=\sigma \frac{\mathrm{d} h^{\prime}}{\mathrm{d} x^{\prime}}
$$

The wave frequency and the wave number are related by the Boussinesq linear dispersion relation:

$$
c^{2}=\frac{\omega^{2}}{k^{2}}=\frac{g h}{1+(k h)^{2} / 3}
$$

and at depth $h_{0}$ the dimensionless phase velocity, $c^{\prime}$, and group velocity, $c_{\mathrm{g}}^{\prime}$, are, respectively

$$
c^{\prime}=1 / \sqrt{1+\frac{\sigma^{2}}{3}} \quad \text { and } \quad c_{\mathrm{g}}^{\prime}=c^{\prime}\left(1-\frac{\sigma^{2}}{3+\sigma^{2}}\right)
$$

The scaled counterparts of Equations (2), up to the second order and assuming that $\varepsilon \sim \sigma^{2}$, are

$$
\frac{\partial \zeta^{\prime}}{\partial t^{\prime}}+\frac{\partial}{\partial x^{\prime}}\left[\left(h^{\prime}+\varepsilon \zeta^{\prime}\right) \bar{u}^{\prime}\right]=0
$$


A PETROV-GALERKIN FEM FOR THE BOUSSINESQ WAVE MODEL

and

$$
\frac{\partial \bar{u}^{\prime}}{\partial t^{\prime}}+\varepsilon \bar{u}^{\prime} \frac{\partial \bar{u}^{\prime}}{\partial x^{\prime}}+\frac{\partial \zeta^{\prime}}{\partial x^{\prime}}-\sigma^{2} \frac{\partial}{\partial t^{\prime}}\left[\frac{h^{\prime}}{2} \frac{\partial^{2}}{\partial\left(x^{\prime}\right)^{2}}\left(h^{\prime} \bar{u}^{\prime}\right)-\frac{\left(h^{\prime}\right)^{2}}{6} \frac{\partial^{2} \bar{u}^{\prime}}{\partial\left(x^{\prime}\right)^{2}}\right]=\mathcal{O}\left(\varepsilon^{2}, \varepsilon \sigma^{2}\right)
$$

If a mild slope of the bottom is assumed, such that $h^{\prime}=h^{\prime}\left(x^{*}\right)$ where $x^{*}=\beta_{\ell} x^{\prime}$, and $\beta_{\ell}$ is a small parameter, then

$$
\frac{\mathrm{d} h^{\prime}}{\mathrm{d} x^{\prime}}=\beta_{\ell} \frac{\mathrm{d} h^{\prime}}{\mathrm{d} x^{*}} \quad \text { and } \quad \frac{\mathrm{d}^{2} h^{\prime}}{\mathrm{d}\left(x^{\prime}\right)^{2}}=\beta_{\ell}^{2} \frac{\mathrm{d}^{2} h^{\prime}}{\mathrm{d}\left(x^{*}\right)^{2}}
$$

For $\beta_{\ell} \sim \sigma$, one has that $\mathrm{d} h / \mathrm{d} x \sim \sigma^{2}$, and the curvature terms and the product of bottom gradient terms in Equation (8b) can be ignored, and thus the equation becomes

$$
\frac{\partial \bar{u}^{\prime}}{\partial t^{\prime}}+\varepsilon \bar{u}^{\prime} \frac{\partial \bar{u}^{\prime}}{\partial x^{\prime}}+\frac{\partial \zeta^{\prime}}{\partial x^{\prime}}-\sigma^{2}\left[\frac{\partial}{\partial x^{\prime}}\left(\frac{\left(h^{\prime}\right)^{2}}{3} \frac{\partial^{2} \bar{u}^{\prime}}{\partial x^{\prime} \partial t^{\prime}}\right)+\beta_{\ell} \frac{h^{\prime}}{3} \frac{\mathrm{d} h^{\prime}}{\mathrm{d} x^{*}} \frac{\partial^{2} \bar{u}^{\prime}}{\partial x^{\prime} \partial t^{\prime}}\right]=\mathcal{O}\left(\varepsilon^{2}, \varepsilon \sigma^{2}, \sigma^{2} \beta_{\ell}^{2}\right)
$$

For an even milder bottom slope, $\beta_{\ell} \sim \varepsilon \sim \sigma^{2}$, one has that $\mathrm{d} h / \mathrm{d} x \sim \sigma^{3}$, and the remaining slopedependent dispersive term can be discarded and the momentum equation will read

$$
\frac{\partial \bar{u}^{\prime}}{\partial t^{\prime}}+\varepsilon \bar{u}^{\prime} \frac{\partial \bar{u}^{\prime}}{\partial x^{\prime}}+\frac{\partial \zeta^{\prime}}{\partial x^{\prime}}-\sigma^{2} \frac{\partial}{\partial x^{\prime}}\left(\frac{\left(h^{\prime}\right)^{2}}{3} \frac{\partial^{2} \bar{u}^{\prime}}{\partial x^{\prime} \partial t^{\prime}}\right)=\mathcal{O}\left(\varepsilon^{2}, \varepsilon \sigma^{2}, \sigma^{2} \beta_{\ell}\right)
$$

The dimensional counterparts of these equations are

- for the mild bottom curvature

$$
\frac{\partial \bar{u}}{\partial t}+\bar{u} \frac{\partial \bar{u}}{\partial x}+g \frac{\partial \zeta}{\partial x}-\frac{\partial}{\partial x}\left(\frac{h^{2}}{3} \frac{\partial^{2} \bar{u}}{\partial x \partial t}\right)-\frac{h}{3} \frac{\mathrm{d} h}{\mathrm{~d} x} \frac{\partial^{2} \bar{u}}{\partial x \partial t}=0
$$

- and for the very mild bottom slope

$$
\frac{\partial \bar{u}}{\partial t}+\bar{u} \frac{\partial \bar{u}}{\partial x}+g \frac{\partial \zeta}{\partial x}-\frac{\partial}{\partial x}\left(\frac{h^{2}}{3} \frac{\partial^{2} \bar{u}}{\partial x \partial t}\right)=0
$$

\subsection{Energy conservation}

This Boussinesq model is not energy conservative [41]. An attempt at writing an energy conservation statement for the very mild bottom slope case leads to

$$
\frac{\partial E^{\prime}}{\partial t^{\prime}}+\frac{\partial F^{\prime}}{\partial x^{\prime}}=-\sigma^{2}\left[\bar{u}^{\prime} \frac{\partial}{\partial x^{\prime}}\left(h^{\prime}+\varepsilon \zeta^{\prime}\right) \frac{\left(h^{\prime}\right)^{2}}{3} \frac{\partial^{2} \bar{u}^{\prime}}{\partial x^{\prime} \partial t^{\prime}}-\varepsilon \frac{\left(h^{\prime}\right)^{2}}{6} \frac{\partial \zeta^{\prime}}{\partial t^{\prime}}\left(\frac{\partial \bar{u}^{\prime}}{\partial x^{\prime}}\right)^{2}\right]
$$

where the energy density is approximated up to $\mathcal{O}\left(\varepsilon^{2} \sigma^{2}\right)$ as

$$
E^{\prime}=\frac{1}{2}\left[\left(\zeta^{\prime}\right)^{2}+\left(h^{\prime}+\varepsilon \zeta^{\prime}\right)\left(\bar{u}^{\prime}\right)^{2}+\sigma^{2}\left(h^{\prime}+\varepsilon \zeta^{\prime}\right) \frac{\left(h^{\prime}\right)^{2}}{3}\left(\frac{\partial \bar{u}^{\prime}}{\partial x^{\prime}}\right)^{2}\right]
$$


and the flux is

$$
F^{\prime}=\left(h^{\prime}+\varepsilon \zeta^{\prime}\right) \bar{u}^{\prime}\left(\zeta^{\prime}+\varepsilon \frac{\left(\bar{u}^{\prime}\right)^{2}}{2}-\sigma^{2} \frac{\left(h^{\prime}\right)^{2}}{3} \frac{\partial^{2} \bar{u}^{\prime}}{\partial x^{\prime} \partial t^{\prime}}\right)
$$

It is evident from (12a) that energy conservation only exists for the non-dispersive case, i.e. for $\sigma=0$.

\section{THE PETROV-GALERKIN SPACE-TIME SCHEME}

Let us consider the domain $Q=\Omega \times T$ where $\Omega=[0, L]$ is the spatial domain of interest and $T=[0,+\infty[$ is the time domain. We shall consider a partition of $Q$ into finite elements in both the space and time directions. Let $\mathscr{V}$ be a finite element space consisting of continuous piecewise bilinear polynomials, and let $\mathscr{S}$ be a finite element space consisting of discontinuous piecewise linear in space and quadratic in time polynomials. The PG formulation of a wave problem described by the Boussinesq equations for mild bottom curvature, Equations (2a) and (11a), will be: find $\hat{\mathbf{U}}=(\hat{\zeta}, \hat{u})^{\mathrm{T}} \in \mathscr{V}^{2}$, such that for all $\Psi(x, t) \in \mathscr{S}^{2}$, one has

$$
\int_{t}^{t+\Delta t} \int_{0}^{L} \boldsymbol{\Psi}^{\mathrm{T}}\left[\frac{\partial \hat{\mathbf{U}}}{\partial t}+\mathbf{A} \frac{\partial \hat{\mathbf{U}}}{\partial x}+\frac{\partial}{\partial x}\left(\mathbf{B} \frac{\partial^{2} \hat{\mathbf{U}}}{\partial x \partial t}\right)+\mathbf{D} \frac{\partial^{2} \hat{\mathbf{U}}}{\partial x \partial t}+\mathbf{C} \hat{\mathbf{U}}\right] \mathrm{d} x \mathrm{~d} t=0
$$

where matrices $\mathbf{A}, \mathbf{B}, \mathbf{C}$, and $\mathbf{D}$ are defined as

$$
\mathbf{A}=\left[\begin{array}{cc}
\hat{u} & h+\hat{\zeta} \\
g & \hat{u}
\end{array}\right], \quad \mathbf{B}=\frac{h^{2}}{3}\left[\begin{array}{cc}
0 & 0 \\
0 & -1
\end{array}\right], \quad \mathbf{C}=\frac{\mathrm{d} h}{\mathrm{~d} x}\left[\begin{array}{cc}
0 & 1 \\
0 & 0
\end{array}\right] \quad \text { and } \quad \mathbf{D}=\frac{h}{3} \frac{\mathrm{d} h}{\mathrm{~d} x}\left[\begin{array}{cc}
0 & 0 \\
0 & -1
\end{array}\right]
$$

The approximate solutions $\hat{\zeta}$ and $\hat{u}$ are interpolated within each element (see Figure 2) using

$$
\hat{\zeta}(\bar{x}, \bar{t})=\sum_{i=1}^{4} M_{i}(\bar{x}, \bar{t}) \hat{\zeta}_{i} \quad \text { and } \quad \hat{u}(\bar{x}, \bar{t})=\sum_{i=1}^{4} M_{i}(\bar{x}, \bar{t}) \hat{u}_{i}
$$

where $\hat{\zeta}_{i}$ and $\hat{u}_{i}$ are the element nodal variables, and the $M_{i}$ are the linear in space and linear in time shape functions:

$$
M_{1}=N_{1}(\bar{x})\left(1-\frac{\bar{t}}{\Delta t}\right), \quad M_{2}=N_{2}(\bar{x})\left(1-\frac{\bar{t}}{\Delta t}\right), \quad M_{3}=N_{1}(\bar{x}) \frac{\bar{t}}{\Delta t} \quad \text { and } \quad M_{4}=N_{2}(\bar{x}) \frac{\bar{t}}{\Delta t}
$$

Herein $N_{1}$ and $N_{2}$ are the usual linear in space shape functions:

$$
N_{1}=1-\frac{\bar{x}}{\Delta x} \quad \text { and } \quad N_{2}=\frac{\bar{x}}{\Delta x}
$$

In the above functions, $\bar{x}$ is the local element space coordinate and $\bar{t}$ is the local element time coordinate. The element side lengths are $\Delta x$ and $\Delta t$, respectively, in the $x$-and $t$-directions.

The weighting function $\boldsymbol{\Psi}$ is a discontinuous linear in space and quadratic in time vectorial function defined on each element for nodes 3 and 4 , for which $\bar{t}=\Delta t$, as

$$
\boldsymbol{\Psi}_{i}(x, t)=\boldsymbol{\Phi}_{i}+\alpha \frac{\Delta x}{2 C_{0}} \mathbf{T}_{\alpha}\left(\mathbf{A}^{\ell}\right) \frac{\partial \mathbf{\Phi}_{i}}{\partial x}+\beta \frac{\Delta x \Delta t}{4 C_{0}}\left(\mathbf{A}^{\ell}\right)^{\mathrm{T}} \frac{\partial^{2} \boldsymbol{\Phi}_{i}}{\partial x \partial t}
$$




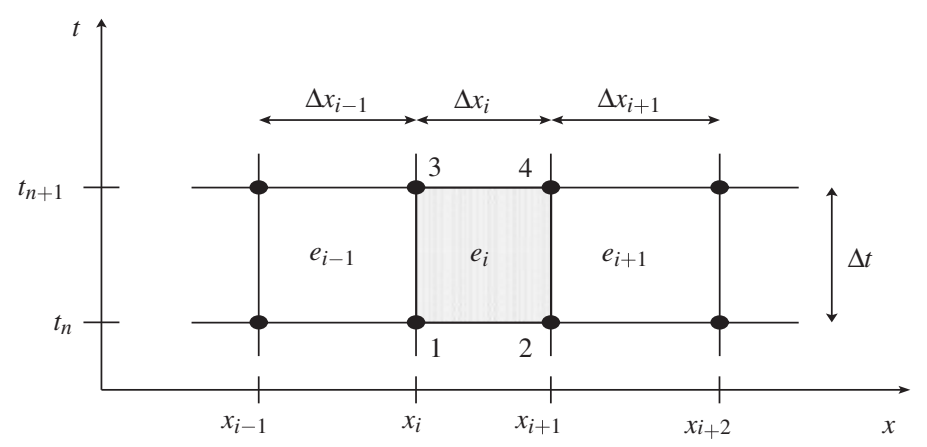

Figure 2. Time-space mesh. Local node numbering at the element $e_{i}$.

where $C_{0}=\sqrt{g h}$ is the asymptotic phase velocity for long waves, and vector $\boldsymbol{\Phi}_{i}$, matrix $\mathbf{A}^{\ell}$, and tensor $\mathbf{T}_{\alpha}$ are, respectively

$$
\boldsymbol{\Phi}_{i}=\left(\varphi_{i}, \varphi_{i}\right)^{\mathrm{T}}, \quad \mathbf{A}^{\ell}=\left[\begin{array}{ll}
0 & h \\
g & 0
\end{array}\right] \quad \text { and } \quad \mathbf{T}_{\alpha}=\left[\begin{array}{ll}
2 & 0 \\
0 & 0
\end{array}\right]
$$

The basis functions $\varphi_{i}$ are

$$
\varphi_{3}=6 N_{1} \frac{\bar{t}}{\Delta t}\left(1-\frac{\bar{t}}{\Delta t}\right) \quad \text { and } \quad \varphi_{4}=6 N_{2} \frac{\bar{t}}{\Delta t}\left(1-\frac{\bar{t}}{\Delta t}\right)
$$

These weighting functions were first proposed in the context of the convective-diffusion equation [40]. The basis functions $\varphi_{3}$ and $\varphi_{4}$, and their derivatives, are shown in Figure 3.

The scalar coefficient $\beta$ must be such that low-order numerical dispersion effects are corrected, while the coefficient $\alpha$ must introduce a high-order dissipation mechanism in the system, in order to guarantee the stability of the numerical scheme. Tensor $\mathbf{T}_{\alpha}$ is necessary in order to prevent the presence of any numerical dissipation term in the continuity equation, which could lead to some loss of mass.

Expanding the weighting function in statement (13) and after integration by parts to reduce the integration order of the unknown functions, one gets that

$$
\begin{gathered}
\int_{t}^{t+\Delta t} \int_{0}^{L}\left[\boldsymbol{\Phi}^{\mathrm{T}} \frac{\partial \hat{\mathbf{U}}}{\partial t}+\alpha \frac{\Delta x}{2 C_{0}}\left(\frac{\partial \boldsymbol{\Phi}}{\partial x}\right)^{\mathrm{T}} \mathbf{A}^{\ell} \mathbf{T}_{\alpha}^{\mathrm{T}} \frac{\partial \hat{\mathbf{U}}}{\partial t}+\beta \frac{\Delta x \Delta t}{4 C_{0}}\left(\frac{\partial^{2} \boldsymbol{\Phi}}{\partial x \partial t}\right)^{\mathrm{T}} \mathbf{A}^{\ell} \frac{\partial \hat{\mathbf{U}}}{\partial t}\right. \\
+\boldsymbol{\Phi}^{\mathrm{T}} \mathbf{A} \frac{\partial \hat{\mathbf{U}}}{\partial x}+\alpha \frac{\Delta x}{2 C_{0}}\left(\frac{\partial \boldsymbol{\Phi}}{\partial x}\right)^{\mathrm{T}} \mathbf{A}^{\ell} \mathbf{T}_{\alpha}^{\mathrm{T}} \mathbf{A} \frac{\partial \hat{\mathbf{U}}}{\partial x}+\beta \frac{\Delta x \Delta t}{4 C_{0}}\left(\frac{\partial^{2} \boldsymbol{\Phi}}{\partial x \partial t}\right)^{\mathrm{T}} \mathbf{A}^{\ell} \mathbf{A} \frac{\partial \hat{\mathbf{U}}}{\partial x} \\
-\frac{\partial \boldsymbol{\Phi}^{\mathrm{T}}}{\partial x} \mathbf{B} \frac{\partial^{2} \hat{\mathbf{U}}}{\partial x \partial t}+\alpha \frac{\Delta x}{2 C_{0}}\left(\frac{\partial \boldsymbol{\Phi}}{\partial x}\right)^{\mathrm{T}} \mathbf{A}^{\ell} \mathbf{T}_{\alpha}^{\mathrm{T}} \frac{\partial}{\partial x}\left(\mathbf{B} \frac{\partial^{2} \hat{\mathbf{U}}}{\partial x \partial t}\right) \\
-\beta \frac{\Delta x \Delta t}{4 C_{0}}\left(\frac{\partial^{3} \boldsymbol{\Phi}}{\partial x^{2} \partial t}\right)^{\mathrm{T}} \mathbf{A}^{\ell} \mathbf{B} \frac{\partial^{2} \hat{\mathbf{U}}}{\partial x \partial t}-\beta \frac{\Delta x \Delta t}{4 C_{0}}\left(\frac{\partial^{2} \mathbf{\Phi}}{\partial x \partial t}\right)^{\mathrm{T}} \frac{\partial}{\partial x}\left(\mathbf{A}^{\ell}\right) \mathbf{B} \frac{\partial^{2} \hat{\mathbf{U}}}{\partial x \partial t}
\end{gathered}
$$



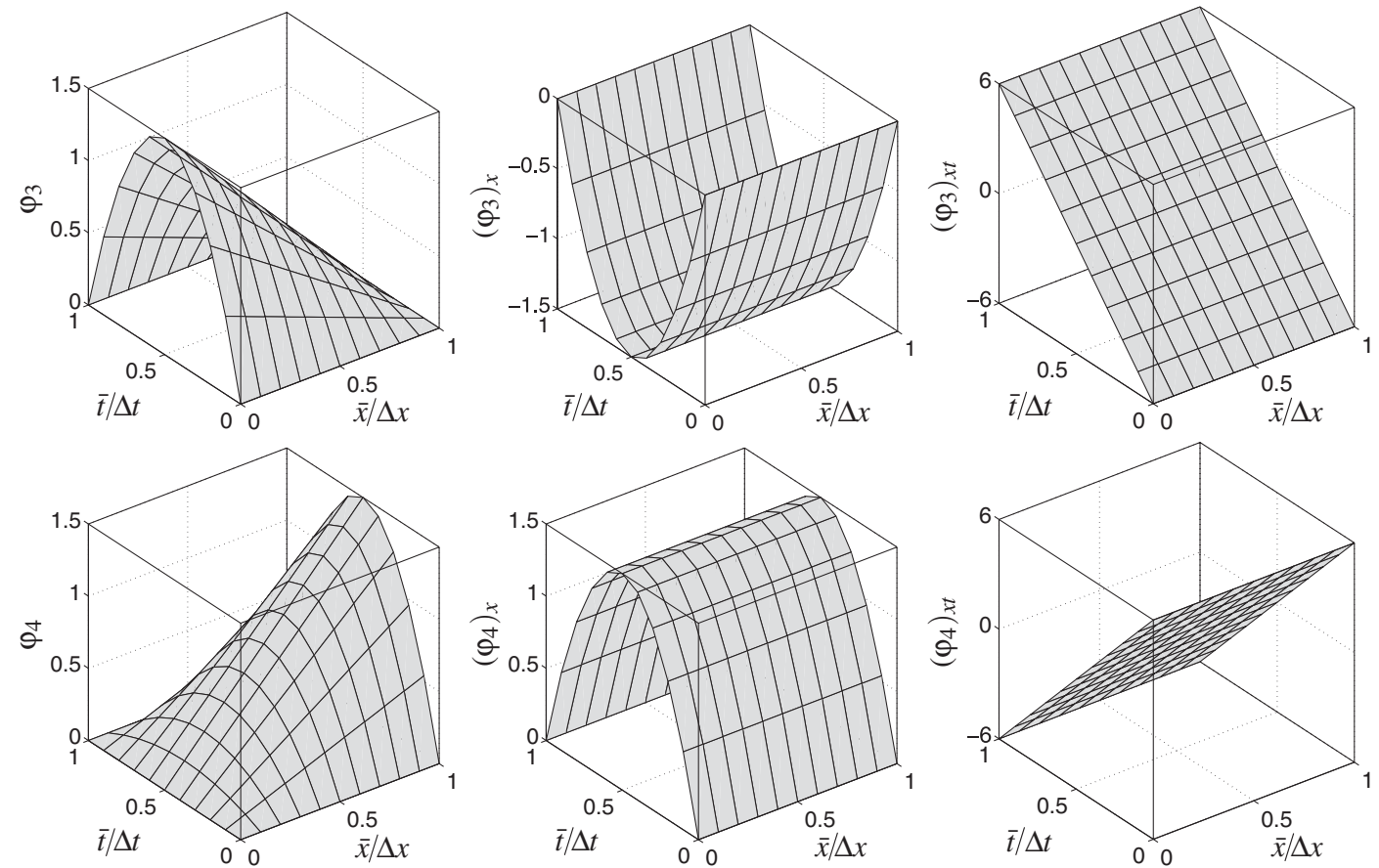

Figure 3. Basis functions $\varphi_{3}$ and $\varphi_{4}$, and their derivatives.

$$
\begin{aligned}
& -\boldsymbol{\Phi}^{\mathrm{T}} \mathbf{D} \frac{\partial^{2} \hat{\mathbf{U}}}{\partial x \partial t}+\alpha \frac{\Delta x}{2 C_{0}}\left(\frac{\partial \boldsymbol{\Phi}}{\partial x}\right)^{\mathrm{T}} \mathbf{A}^{\ell} \mathbf{T}_{\alpha}^{\mathrm{T}} \mathbf{D} \frac{\partial^{2} \hat{\mathbf{U}}}{\partial x \partial t}+\beta \frac{\Delta x \Delta t}{4 C_{0}}\left(\frac{\partial^{2} \boldsymbol{\Phi}}{\partial x \partial t}\right)^{\mathrm{T}} \mathbf{A}^{\ell} \mathbf{D} \frac{\partial^{2} \hat{\mathbf{U}}}{\partial x \partial t} \\
& \left.+\boldsymbol{\Phi}^{\mathrm{T}} \mathbf{C} \mathbf{U}+\alpha \frac{\Delta x}{2 C_{0}}\left(\frac{\partial \boldsymbol{\Phi}}{\partial x}\right)^{\mathrm{T}} \mathbf{A}^{\ell} \mathbf{T}_{\alpha}^{\mathrm{T}} \mathbf{C} \mathbf{U}+\beta \frac{\Delta x \Delta t}{4 C_{0}}\left(\frac{\partial^{2} \boldsymbol{\Phi}}{\partial x \partial t}\right)^{\mathrm{T}} \mathbf{A}^{\ell} \mathbf{C} \mathbf{U}\right] \mathrm{d} x \mathrm{~d} t \\
& +\int_{t}^{t+\Delta t}\left[\boldsymbol{\Phi}^{\mathrm{T}} \mathbf{B} \frac{\partial^{2} \hat{\mathbf{U}}}{\partial x \partial t}+\beta \frac{\Delta x \Delta t}{4 C_{0}}\left(\frac{\partial^{2} \boldsymbol{\Phi}}{\partial x \partial t}\right)^{\mathrm{T}} \mathbf{A}^{\ell} \mathbf{B} \frac{\partial^{2} \hat{\mathbf{U}}}{\partial x \partial t}\right]_{0}^{L} \mathrm{~d} t=0 \quad \forall \boldsymbol{\Phi}
\end{aligned}
$$

This statement can be re-written as a system of two scalar equations becoming: find functions $\hat{\zeta}$ and $\hat{u}$ such that

$$
\begin{aligned}
& \int_{t}^{t+\Delta t} \int_{0}^{L}\left(\hat{\varphi} \frac{\partial \hat{\zeta}}{\partial t}+\hat{\varphi} \frac{\partial \hat{p}}{\partial x}\right) \mathrm{d} x \mathrm{~d} t \\
& +\int_{t}^{t+\Delta t} \int_{0}^{L} \beta \frac{\Delta x \Delta t}{4 C_{0}}\left(h \frac{\partial^{2} \hat{\varphi}}{\partial x \partial t} \frac{\partial \hat{q}}{\partial x}+h \frac{\partial^{2} \hat{\varphi}}{\partial x \partial t} \frac{\partial \hat{u}}{\partial t}+\frac{h^{3}}{3} \frac{\partial^{3} \hat{\varphi}}{\partial x^{2} \partial t} \frac{\partial^{2} \hat{u}}{\partial x \partial t}\right) \mathrm{d} x \mathrm{~d} t \\
& +\int_{t}^{t+\Delta t}\left[\beta \frac{\Delta x \Delta t}{4 C_{0}} \frac{h^{3}}{3} \frac{\partial^{2} \hat{\varphi}}{\partial x \partial t} \frac{\partial^{2} \hat{u}}{\partial x \partial t}\right]_{0}^{L} \mathrm{~d} t=0 \quad \forall \hat{\varphi}
\end{aligned}
$$


and

$$
\begin{aligned}
& \int_{t}^{t+\Delta t} \int_{0}^{L}\left(\hat{\varphi} \frac{\partial \hat{u}}{\partial t}+\hat{\varphi} \frac{\partial \hat{q}}{\partial x}+\frac{h^{2}}{3} \frac{\partial \hat{\varphi}}{\partial x} \frac{\partial^{2} \hat{u}}{\partial x \partial t}-\frac{h}{3} \frac{\partial h}{\partial x} \hat{\varphi} \frac{\partial^{2} \hat{u}}{\partial x \partial t}\right) \mathrm{d} x \mathrm{~d} t \\
& +\int_{t}^{t+\Delta t} \int_{0}^{L} \alpha \frac{\Delta x}{C_{0}} g\left(\frac{\partial \hat{\varphi}}{\partial x} \frac{\partial \hat{\zeta}}{\partial t}+\frac{\partial \hat{\varphi}}{\partial x} \frac{\partial \hat{p}}{\partial x}\right) \mathrm{d} x \mathrm{~d} t \\
& +\int_{t}^{t+\Delta t} \int_{0}^{L} \beta \frac{\Delta x \Delta t}{4 C_{0}} g \frac{\partial^{2} \hat{\varphi}}{\partial x \partial t} \frac{\partial \hat{p}}{\partial x} \mathrm{~d} x \mathrm{~d} t-\int_{t}^{t+\Delta t}\left[\varphi \frac{h^{2}}{3} \frac{\partial^{2} \hat{u}}{\partial x \partial t}\right]_{0}^{L} \mathrm{~d} t=0 \quad \forall \hat{\varphi}
\end{aligned}
$$

where $\hat{p}$ and $\hat{q}$ are, respectively, the depth-integrated local horizontal momentum density and the local wave energy density:

$$
\hat{p}=(h+\hat{\zeta}) \hat{u} \quad \text { and } \quad \hat{q}=\frac{\hat{u}^{2}}{2}+g \hat{\zeta}
$$

Within each element these quantities are approximated as

$$
\hat{p}=\sum_{i=1}^{4} M_{i}\left(h_{i}+\hat{\zeta}_{i}\right) \hat{u}_{i} \quad \text { and } \quad \hat{q}=\sum_{i=1}^{4} M_{i}\left(\frac{\hat{u}_{i}^{2}}{2}+g \hat{\zeta}_{i}\right)
$$

The second and third terms in the second integral in Equation (22a) and the third integral in the same equation can be disposed of in at least two ways of reasoning:

(a) when depth is constant in time, the integral of the product of two time derivatives of the shape and weighting functions, $M_{i}$ and $\varphi_{i}$, will always be null;

(b) if $\Delta x \sim \Delta t \sim \sigma$, then the terms in $h^{3} / 3$ will be at least $\mathcal{O}\left(\sigma^{4}\right)$. Hence, they are of the same order of magnitude of the analytical error of the Boussinesq equations (8a) and (10a).

Therefore, we can replace Equation (22a) by

$$
\int_{t}^{t+\Delta t} \int_{0}^{L}\left(\hat{\varphi} \frac{\partial \hat{\zeta}}{\partial t}+\hat{\varphi} \frac{\partial \hat{p}}{\partial x}\right) \mathrm{d} x \mathrm{~d} t+\int_{t}^{t+\Delta t} \int_{0}^{L} \beta \frac{\Delta x \Delta t}{4 C_{0}} h \frac{\partial^{2} \hat{\varphi}}{\partial x \partial t} \frac{\partial \hat{q}}{\partial x} \mathrm{~d} x \mathrm{~d} t=0 \quad \forall \hat{\varphi}
$$

Let us now discretize Equations (22b) and (22c) by means of the element shape functions (16) and of the weighting functions (20). The original set of partial differential equations may now be recast as a set of algebraic equations. We chose to consider the non-linear term, the bottom gradient term, and the $\alpha$ - and $\beta$-terms, as forcing terms. In this manner, the system matrices are linear, symmetric, and time independent. In a matrix-like fashion and neglecting the contribution of the boundary terms we may write that

$$
\begin{aligned}
\mathbf{M} \Delta \zeta= & -\frac{\Delta t}{2} \mathbf{C}\left(\mathbf{p}^{(n+1)}+\mathbf{p}^{(n)}\right)+\beta \frac{\Delta x \Delta t}{4 C_{0}} \mathbf{K}_{h} \Delta \mathbf{q} \\
\left(\mathbf{M}+\mathbf{K}_{\sigma}\right) \Delta \mathbf{u}= & -\frac{\Delta t}{2}\left[\mathbf{C}\left(\mathbf{q}^{(n+1)}+\mathbf{q}^{(n)}\right)+\alpha \frac{\Delta x}{C_{0}} g \mathbf{K}\left(\mathbf{p}^{(n+1)}+\mathbf{p}^{(n)}\right)\right] \\
& +\mathbf{C}_{h} \Delta \mathbf{u}-\alpha \frac{\Delta x}{C_{0}} g \mathbf{C}^{\mathrm{T}} \Delta \zeta+\beta \frac{\Delta x \Delta t}{4 C_{0}} g \mathbf{K} \Delta \mathbf{p}
\end{aligned}
$$




\section{P. AVILEZ-VALENTE AND F. J. SEABRA-SANTOS}

Herein $\zeta^{(n)}, \mathbf{u}^{(n)}, \mathbf{p}^{(n)}$, and $\mathbf{q}^{(n)}$ stand for the nodal value vectors for functions $\hat{\zeta}, \hat{u}, \hat{p}$, and $\hat{q}$, respectively, computed at the time level $t_{n}$. The vectors of nodal increments, $\Delta \zeta$ and $\Delta \mathbf{u}$, are

$$
\Delta \zeta=\zeta^{(n+1)}-\zeta^{(n)} \text { and } \Delta \mathbf{u}=\mathbf{u}^{(n+1)}-\mathbf{u}^{(n)}
$$

$\mathbf{M}, \mathbf{C}$, and $\mathbf{K}$ are the usual element mass, convection, and diffusion matrices with entries, respectively,

$$
M_{i j}=\left\langle N_{i} N_{j}\right\rangle, \quad C_{i j}=\left\langle N_{i} \frac{\mathrm{d} N_{j}}{\mathrm{~d} x}\right\rangle \quad \text { and } \quad K_{i j}=\left\langle\frac{\mathrm{d} N_{i}}{\mathrm{~d} x} \frac{\mathrm{d} N_{j}}{\mathrm{~d} x}\right\rangle
$$

where $\langle\cdot\rangle$ stands for $\int_{x_{j}}^{x_{j+1}}(\cdot) \mathrm{d} x$. Matrices $\mathbf{K}_{h}, \mathbf{K}_{\sigma}$, and $\mathbf{C}_{h}$ have entries

$$
\begin{aligned}
& \left(K_{h}\right)_{i j}=\sum_{k=1}^{2}\left\langle\frac{\mathrm{d} N_{i}}{\mathrm{~d} x} N_{k} \frac{\mathrm{d} N_{j}}{\mathrm{~d} x}\right\rangle h_{k} \\
& \left(K_{\sigma}\right)_{i j}=\frac{1}{3} \sum_{k=1}^{2} \sum_{\ell=1}^{2}\left\langle\frac{\mathrm{d} N_{i}}{\mathrm{~d} x} N_{k} N_{\ell} \frac{\mathrm{d} N_{j}}{\mathrm{~d} x}\right\rangle h_{k} h_{\ell} \\
& \left(C_{h}\right)_{i j}=\frac{1}{3} \sum_{k=1}^{2} \sum_{\ell=1}^{2}\left\langle N_{i} N_{k} \frac{\mathrm{d} N_{\ell}}{\mathrm{d} x} \frac{\mathrm{d} N_{j}}{\mathrm{~d} x}\right\rangle h_{k} h_{\ell}
\end{aligned}
$$

In Equations (23), $C_{0}, \alpha$, and $\beta$ are computed assuming a constant mean depth over the element length.

\subsection{Predictor-corrector}

Having applied a bilinear FEM for the space and time discretization, an integration over each time slab is necessary. This is essentially equivalent to a one-step time integration scheme. Nevertheless, the non-linear nature of the Boussinesq equations still requires an iterative solution for this integration.

We propose a predictor-corrector scheme with two or three corrector steps. As we will show later, the optimal values for the coefficients $\alpha$ and $\beta$ will change at each step of this inner iteration.

Predictor step: Assuming that $\mathbf{p}^{(n+1)}=\mathbf{p}^{(n)}$ and $\mathbf{q}^{(n+1)}=\mathbf{q}^{(n)}$, and that $\mathbf{u}^{(n+1)}=\mathbf{u}^{(n)}$ in the slope induced term, the set of algebraic equations (23) may be recast as

$$
\begin{gathered}
\mathbf{M} \Delta \zeta_{0}=-\Delta t \mathbf{C p}^{(n)} \\
\left(\mathbf{M}+\mathbf{K}_{\sigma}\right) \Delta \mathbf{u}_{0}=-\Delta t\left(\mathbf{C} \mathbf{q}^{(n)}+\alpha_{0} \frac{\Delta x}{C_{0}} g \mathbf{K} \mathbf{p}^{(n)}\right)
\end{gathered}
$$

The vectors of nodal increments, $\Delta \zeta_{0}$ and $\Delta \mathbf{u}_{0}$, are, respectively,

$$
\Delta \zeta_{0}=\zeta_{0}^{(n+1)}-\zeta^{(n)} \text { and } \quad \Delta \mathbf{u}_{0}=\mathbf{u}_{0}^{(n+1)}-\mathbf{u}^{(n)}
$$

The subscript ' 0 ' indicates values computed at the predictor step. 
A PETROV-GALERKIN FEM FOR THE BOUSSINESQ WAVE MODEL

Corrector steps: The system of equations to be solved at the $k$ th corrector step are

$$
\begin{aligned}
\mathbf{M} \Delta \zeta_{k}=- & \frac{\Delta t}{2} \mathbf{C}\left(\mathbf{p}_{k-1}^{(n+1)}+\mathbf{p}^{(n)}\right)+\beta_{k} \frac{\Delta x \Delta t}{4 C_{0}} \mathbf{K}_{h} \Delta \mathbf{q}_{k-1} \\
\left(\mathbf{M}+\mathbf{K}_{\sigma}\right) \Delta \mathbf{u}_{k}= & -\frac{\Delta t}{2}\left[\mathbf{C}\left(\mathbf{q}_{k-1}^{(n+1)}+\mathbf{q}^{(n)}\right)+\alpha_{k} \frac{\Delta x}{C_{0}} g \mathbf{K}\left(\mathbf{p}_{k-1}^{(n+1)}+\mathbf{p}^{(n)}\right)\right] \\
& +\mathbf{C}_{h} \Delta \mathbf{u}_{k-1}-\alpha_{k} \frac{\Delta x}{C_{0}} g \mathbf{C}^{\mathrm{T}} \Delta \zeta_{k-1}+\beta_{k} \frac{\Delta x \Delta t}{4 C_{0}} g \mathbf{K} \Delta \mathbf{p}_{k-1}
\end{aligned}
$$

where

$$
\Delta \zeta_{k}=\zeta_{k}^{(n+1)}-\zeta^{(n)} \quad \text { and } \quad \Delta \mathbf{u}_{k}=\mathbf{u}_{k}^{(n+1)}-\mathbf{u}^{(n)}
$$

The subscript ' $k$ ' denotes values computed at the $k$ th corrector step.

\section{ACCURACY AND STABILITY ANALYSIS}

The accuracy and stability of the proposed PG FEM depend on the coefficients $\alpha$ and $\beta$ on the weighting functions. The values these coefficients shall assume will depend on the inner iterations strategy adopted to deal with the non-linear term. We shall begin by analysing the truncation error of the non-linear system of discrete equations (23) by means of the equivalent differential equation method (see Reference [42, Chapter 9]). Later we shall resort to a von Neumann analysis in the spectral space (see Reference [42, Chapter 8]) to establish the minimal number of corrector steps to be performed during the iterative solution within each time-slab.

\subsection{Non-linear analysis}

Let us write the non-dimensional counterpart of Equations (23), assuming a constant depth $h_{0}$, on a regular space-time finite element mesh, in what follows and for the sake of brevity, the prime will be dropped:

$$
\begin{gathered}
\mathbf{M} \Delta \zeta=-\frac{\Delta t}{2} \mathbf{C}\left(\mathbf{p}^{(n+1)}+\mathbf{p}^{(n)}\right)+\beta \frac{\Delta x \Delta t}{4} \mathbf{K} \Delta \mathbf{q} \\
\left(\mathbf{M}+\frac{\sigma^{2}}{3} \mathbf{K}\right) \Delta \mathbf{u}=-\frac{\Delta t}{2}\left[\mathbf{C}\left(\mathbf{q}^{(n+1)}+\mathbf{q}^{(n)}\right)+\alpha \Delta x \mathbf{K}\left(\mathbf{p}^{(n+1)}+\mathbf{p}^{(n)}\right)\right] \\
-\alpha \Delta x \mathbf{C}^{\mathrm{T}} \Delta \zeta+\beta \frac{\Delta x \Delta t}{4} \mathbf{K} \Delta \mathbf{p}
\end{gathered}
$$

In Equations (31), the dimensionless momentum and wave energy densities are

$$
\hat{p}=(1+\varepsilon \hat{\zeta}) \hat{u} \quad \text { and } \quad \hat{q}=\varepsilon \frac{\hat{u}^{2}}{2}+\hat{\zeta}
$$




\section{P. AVILEZ-VALENTE AND F. J. SEABRA-SANTOS}

Assembling Equations (31) over the entire computational domain leads us to the following discrete equations for the $i$ th node, away from the boundaries:

$$
\begin{aligned}
\mathscr{L}_{\zeta} \equiv & \frac{\Delta x}{6}\left[\left(\hat{\zeta}_{i+1}^{(n+1)}+4 \hat{\zeta}_{i}^{(n+1)}+\hat{\zeta}_{i-1}^{(n+1)}\right)-\left(\hat{\zeta}_{i+1}^{(n)}+4 \hat{\zeta}_{i}^{(n)}+\hat{\zeta}_{i-1}^{(n)}\right)\right] \\
& +\frac{\Delta t}{4}\left\{\left[\left(1+\varepsilon \hat{\zeta}_{i+1}^{(n+1)}\right) \hat{u}_{i+1}^{(n+1)}-\left(1+\varepsilon \hat{\zeta}_{i-1}^{(n+1)}\right) \hat{u}_{i-1}^{(n+1)}\right]\right. \\
& \left.+\left[\left(1+\varepsilon \hat{\zeta}_{i+1}^{(n)}\right) \hat{u}_{i+1}^{(n)}-\left(1+\varepsilon \hat{\zeta}_{i-1}^{(n)}\right) \hat{u}_{i-1}^{(n)}\right]\right\} \\
& +\varepsilon \beta \frac{\Delta t}{8}\left\{\left[\left(\hat{u}_{i+1}^{(n+1)}\right)^{2}-2\left(\hat{u}_{i}^{(n+1)}\right)^{2}+\left(\hat{u}_{i-1}^{(n+1)}\right)^{2}\right]-\left[\left(\hat{u}_{i+1}^{(n)}\right)^{2}-2\left(\hat{u}_{i}^{(n)}\right)^{2}+\left(\hat{u}_{i-1}^{(n)}\right)^{2}\right]\right\} \\
& +\beta \frac{\Delta t}{4}\left[\left(\hat{\zeta}_{i+1}^{(n+1)}-2 \hat{\zeta}_{i}^{(n+1)}+\hat{\zeta}_{i-1}^{(n+1)}\right)-\left(\hat{\zeta}_{i+1}^{(n)}-2 \hat{\zeta}_{i}^{(n)}+\hat{\zeta}_{i-1}^{(n)}\right)\right]=0
\end{aligned}
$$

and

$$
\begin{aligned}
\mathscr{L}_{\bar{u}} \equiv & \frac{\Delta x}{6}\left[\left(\hat{u}_{i+1}^{(n+1)}+4 \hat{u}_{i}^{(n+1)}+\hat{u}_{i-1}^{(n+1)}\right)-\left(\hat{u}_{i+1}^{(n)}+4 \hat{u}_{i}^{(n)}+\hat{u}_{i-1}^{(n)}\right)\right] \\
& +\varepsilon \frac{\Delta t}{4}\left\{\left[\left(\hat{u}_{i+1}^{(n+1)}\right)^{2}-\left(\hat{u}_{i-1}^{(n+1)}\right)^{2}\right]+\left[\left(\hat{u}_{i+1}^{(n)}\right)^{2}-\left(\hat{u}_{i-1}^{(n)}\right)^{2}\right]\right\} \\
& +\frac{\Delta t}{4}\left[\left(\hat{\zeta}_{i+1}^{(n+1)}-\hat{\zeta}_{i-1}^{(n+1)}\right)+\left(\hat{\zeta}_{i+1}^{(n)}-\hat{\zeta}_{i-1}^{(n)}\right)\right] \\
& -\frac{\sigma^{2}}{3 \Delta x}\left[\left(\hat{u}_{i+1}^{(n+1)}-2 \hat{u}_{i}^{(n+1)}+\hat{u}_{i-1}^{(n+1)}\right)-\left(\hat{u}_{i+1}^{(n)}-2 \hat{u}_{i}^{(n)}+\hat{u}_{i-1}^{(n)}\right)\right] \\
& -\alpha \frac{\Delta x}{2}\left[\left(\hat{\zeta}_{i+1}^{(n+1)}-\hat{\zeta}_{i-1}^{(n+1)}\right)-\left(\hat{\zeta}_{i+1}^{(n)}-\hat{\zeta}_{i-1}^{(n)}\right)\right] \\
& -\alpha \frac{\Delta t}{2}\left\{\left[\left(1+\varepsilon \hat{\zeta}_{i+1}^{(n+1)}\right) \hat{u}_{i+1}^{(n+1)}-2\left(1+\varepsilon \hat{\zeta}_{i}^{(n+1)}\right) \hat{u}_{i}^{(n+1)}+\left(1+\varepsilon \hat{\zeta}_{i-1}^{(n+1)}\right) \hat{u}_{i-1}^{(n+1)}\right]\right. \\
& \left.+\left[\left(1+\varepsilon \hat{\zeta}_{i+1}^{(n)}\right) \hat{u}_{i+1}^{(n)}-2\left(1+\varepsilon \hat{\zeta}_{i}^{(n)}\right) \hat{u}_{i}^{(n)}+\left(1+\varepsilon \hat{\zeta}_{i-1}^{(n)}\right) \hat{u}_{i-1}^{(n)}\right]\right\} \\
& +\beta \frac{\Delta t}{4}\left\{\left[\left(1+\varepsilon \hat{\zeta}_{i+1}^{(n+1)}\right) \hat{u}_{i+1}^{(n+1)}-2\left(1+\varepsilon \hat{\zeta}_{i}^{(n+1)}\right) \hat{u}_{i}^{(n+1)}+\left(1+\varepsilon \hat{\zeta}_{i-1}^{(n+1)}\right) \hat{u}_{i-1}^{(n+1)}\right]\right. \\
& \left.-\left[\left(1+\varepsilon \hat{\zeta}_{i+1}^{(n)}\right) \hat{u}_{i+1}^{(n)}-2\left(1+\varepsilon \hat{\zeta}_{i}^{(n)}\right) \hat{u}_{i}^{(n)}+\left(1+\varepsilon \hat{\zeta}_{i-1}^{(n)}\right) \hat{u}_{i-1}^{(n)}\right]\right\}=0
\end{aligned}
$$

where $\hat{\zeta}_{i}^{(n)}=\hat{\zeta}\left(x_{i}, t_{n}\right), \hat{u}_{i}^{(n)}=\hat{u}\left(x_{i}, t_{n}\right)$, and $\left(\hat{u}_{i}^{(n)}\right)^{2}=\left(\hat{u}\left(x_{i}, t_{n}\right)\right)^{2}$.

Owing to the finite element truncation errors, the discrete algebraic operators $\mathscr{L}_{\zeta}$ and $\mathscr{L}_{\bar{u}}$ are indeed better approximations for a set of differential equations other than (8a) and (10b). A new set of equations, the so-called equivalent differential equations of the numerical scheme, can be obtained from the discrete algebraic operators $\mathscr{L}_{\zeta}$ and $\mathscr{L}_{\bar{u}}$, after replacing $\hat{\zeta}_{i+\ell}^{(n+1 / 2+m)}$ and $\hat{u}_{i+\ell}^{(n+1 / 2+m)}$, for $m=-\frac{1}{2}, \frac{1}{2}$ and $\ell=-1,0,1$, by their fifth-order Taylor series approximation around $\hat{\zeta}_{i}^{(n+1 / 2)}$ and $\hat{u}_{i}^{(n+1 / 2)}$, respectively.

This new set of differential equations must be rearranged in order to replace higher time derivatives and/or cross time-space derivatives by equivalent space derivatives, except for $\partial^{3} \hat{u} / \partial x^{2} \partial t$. This can be accomplished through recursive differentiation of the new equations and substitution 
onto themselves. Such a procedure will eventually lead us to the following set of equivalent differential equations of which the proposed numerical scheme is a better approximation:

$$
\frac{\partial \hat{\zeta}}{\partial t}+\frac{\partial}{\partial x}[(1+\varepsilon \hat{\zeta}) \hat{u}]=\frac{1}{4} C r \Delta x^{2}\left(\beta-\frac{C r}{3}\right) \frac{\partial^{3} \hat{u}}{\partial x^{3}}+\mathcal{O}\left(\Delta x^{4}, \varepsilon \Delta x^{2}, \sigma^{2} \Delta x^{2}\right)
$$

and

$$
\begin{aligned}
\frac{\partial \hat{u}}{\partial t}+\varepsilon \hat{u} \frac{\partial \hat{u}}{\partial x}+\frac{\partial \hat{\zeta}}{\partial x}-\frac{\sigma^{2}}{3} \frac{\partial^{3} \hat{u}}{\partial x^{2} \partial t}= & \frac{1}{4} C r \Delta x^{2}\left(\beta-\frac{C r}{3}\right) \frac{\partial^{3} \hat{\zeta}}{\partial x^{3}}-\frac{1}{12} \Delta x^{3} \alpha(1-3 \beta C r) \frac{\partial^{4} \hat{u}}{\partial x^{4}} \\
& +\mathcal{O}\left(\Delta x^{4}, \varepsilon \Delta x^{2}, \sigma^{2} \Delta x^{2}\right)
\end{aligned}
$$

In Equations (34), $\mathrm{Cr}$ is the Courant number, defined as

$$
C r=\frac{\Delta t^{\prime}}{\Delta x^{\prime}} \quad \text { or } \quad C r=C_{0} \frac{\Delta t}{\Delta x}
$$

It could be shown that for $\beta=0$ and $\alpha=0$, the proposed scheme is equivalent to the usual $\mathrm{BG}$ finite element formulation in space, with trial and weighting functions defined in the same space of linear polynomial functions, associated with a Crank-Nicholson finite difference scheme for time integration. Comparison of Equations (34), and Equations (8a) and (10b), shows that for $\beta=0$ and $\alpha=0$ the scheme would be affected by a second-order dispersion error and would possess no dissipative character.

Dispersion correction: The proposed PG formulation can be made fourth-order accurate by setting

$$
\beta=\frac{C r}{3} \quad \text { and } \quad \alpha=0
$$

Such a choice for the coefficients eliminates the second-order numerical terms in Equations (34), and the associated dispersion error, while no dissipation mechanism is added. The equivalent differential equation is now a fourth-order approximation of the Boussinesq equations, provided that $\varepsilon \sim \sigma^{2} \sim \Delta x^{2} \sim \Delta t^{2}$ :

$$
\frac{\partial \hat{\zeta}}{\partial t}+\frac{\partial}{\partial x}[(1+\varepsilon \hat{\zeta}) \hat{u}]=\mathcal{O}\left(\Delta x^{4}, \varepsilon \Delta x^{2}, \sigma^{2} \Delta x^{2}\right)
$$

and

$$
\frac{\partial \hat{u}}{\partial t}+\varepsilon \hat{u} \frac{\partial \hat{u}}{\partial x}+\frac{\partial \hat{\zeta}}{\partial x}-\frac{\sigma^{2}}{3} \frac{\partial^{3} \hat{u}}{\partial x^{2} \partial t}=\mathcal{O}\left(\Delta x^{4}, \varepsilon \Delta x^{2}, \sigma^{2} \Delta x^{2}\right)
$$

Such a formulation, although highly accurate, does not possess any dissipative term, which are associated with odd powers of $\Delta x$. Therefore, some kind of filtering will be necessary for the actual numerical code to be stable.

Stability criterion: Let us assume that $\beta=C r / 3$, and $\alpha \neq 0$. In this case the equivalent differential equations read

$$
\frac{\partial \hat{\zeta}}{\partial t}+\frac{\partial}{\partial x}[(1+\varepsilon \hat{\zeta}) \hat{u}]=\mathcal{O}\left(\Delta x^{4}, \varepsilon \Delta x^{2}, \sigma^{2} \Delta x^{2}\right)
$$


and

$$
\frac{\partial \hat{u}}{\partial t}+\varepsilon \hat{u} \frac{\partial \hat{u}}{\partial x}+\frac{\partial \hat{\zeta}}{\partial x}-\frac{\sigma^{2}}{3} \frac{\partial^{3} \hat{u}}{\partial x^{2} \partial t}=-\frac{1}{12} \Delta x^{3} \alpha\left(1-C r^{2}\right) \frac{\partial^{4} \hat{u}}{\partial x^{4}}+\mathcal{O}\left(\Delta x^{4}, \varepsilon \Delta x^{2}, \sigma^{2} \Delta x^{2}\right)
$$

For the scheme to be stable, the third-order linear term associated with the fourth derivative $\partial^{4} \hat{u} / \partial x^{4}$ in Equation (38b) shall have a negative coefficient. Several expressions could be chosen for $\alpha$ to fulfil this condition. We propose

$$
\alpha=\frac{C r^{3}}{1-C r^{2}} \quad \text { for } C r<1
$$

Hence, the equivalent differential equations become

$$
\frac{\partial \hat{\zeta}}{\partial t}+\frac{\partial}{\partial x}[(1+\varepsilon \hat{\zeta}) \hat{u}]=\mathcal{O}\left(\Delta x^{4}, \varepsilon \Delta x^{2}, \sigma^{2} \Delta x^{2}\right)
$$

and

$$
\frac{\partial \hat{u}}{\partial t}+\varepsilon \hat{u} \frac{\partial \hat{u}}{\partial x}+\frac{\partial \hat{\zeta}}{\partial x}-\frac{\sigma^{2}}{3} \frac{\partial^{3} \hat{u}}{\partial x^{2} \partial t}=-\frac{1}{12} C r^{3} \Delta x^{3} \frac{\partial^{4} \hat{u}}{\partial x^{4}}+\mathcal{O}\left(\Delta x^{4}, \varepsilon \Delta x^{2}, \sigma^{2} \Delta x^{2}\right)
$$

Clearly for stability to be attained the Courant number shall be smaller than 1 . Nevertheless, nothing can be said on the higher-order terms that will also be a function of $\alpha$ and $\beta$. Their behaviour shall be investigated by means of a linear analysis in the spectral space.

\subsection{Linear analysis}

The equivalent differential equation method, although applicable to non-linear differential equations, is not convenient when a higher-order analysis is sought. For that purpose, a von Neumann analysis on the spectral space will be used. This analysis is restricted to the linearized equations.

Let us assume that a plane wave solution of the linearized, i.e. $\varepsilon=0$, version of the discrete equations (31), at the time instant $t_{n}=n \Delta t$ and at the $j$ th node, can be expressed by the individual Fourier components:

$$
\left\{\begin{array}{l}
\hat{\zeta}_{j}^{(n)}=G^{n} \hat{\zeta}_{0}^{(0)} \mathrm{e}^{\hat{\imath}(j \Delta x)} \\
\hat{u}_{j}^{(n)}=G^{n} \hat{u}_{0}^{(0)} \mathrm{e}^{\hat{\imath}(j \Delta x)}
\end{array}\right.
$$

Herein, $G$ is a complex propagation factor defined as

$$
G=\mathrm{e}^{-(\hat{\imath} \hat{c}+\hat{s}) \Delta t}
$$

where $\hat{c}$ and $\hat{s}$ are the linear phase velocity and the damping ratio of the numerical wave, respectively. Under such conditions, a linear scheme is neutrally stable if $|G|=1$, stable and dissipative if $|G|<1$, and unstable if $|G|>1$.

The complex propagation factor is computed by replacing expressions (41) into the linear version of Equations (31) and algebraically solving the resulting eigenvalue problem for $G$. Two distinct 
complex conjugate modes, $G_{1}$ and $G_{2}$, are obtained which correspond to two waves propagating in opposite directions:

$$
G=\frac{A \sigma^{2}+B \Delta x^{2} \pm 6 \sqrt{C \Delta x^{2} \sigma^{2}+\left(F+D^{2} \alpha^{2}\right) \Delta x^{4}}}{A \sigma^{2}+(H-6 D \alpha) \Delta x^{2}}
$$

where

$$
\begin{gathered}
E=3 \beta C r(1-\cos \Delta x)-2(1+\cos \Delta x)-2 \\
A=4 E(1-\cos \Delta x) \\
B=4 E-9 C r^{2} \beta^{2}(1-\cos \Delta x)^{2}+3 C r(4 \beta+3 C r) \sin \Delta x^{2}-4(1+\cos \Delta x)^{2}+4 \\
C=4 E C r^{2} \sin ^{2} \Delta x(1-\cos \Delta x) \\
D=C r(1-3 \beta C r)(1-\cos \Delta x)^{2} \\
F=-C r^{2} \sin ^{2} \Delta x E^{2} \\
H=4 E-9 C r^{2} \beta^{2}(1-\cos \Delta x)^{2}+3 C r(4 \beta-3 C r) \sin \Delta x^{2}-4(1+\cos \Delta x)^{2}+4
\end{gathered}
$$

The phase velocity and the damping ratio of the numerical wave can be computed from $G$ as

$$
\begin{gathered}
\hat{c}=-\operatorname{Im}\left[\frac{1}{C r \Delta x} \log G\right] \quad \text { or } \quad \hat{c}=\frac{1}{C r \Delta x} \arctan \left(\frac{-\operatorname{Im} G}{\operatorname{Re} G}\right) \\
\hat{s}=\operatorname{Re}\left[\frac{1}{C r \Delta x} \log G\right]
\end{gathered}
$$

where Re and Im stand for the real and imaginary parts of a complex number, respectively. The numerical group velocity, $\hat{c}_{\mathrm{g}}$, is given by

$$
\hat{c}_{\mathrm{g}}=\hat{c}+\sigma \frac{\partial \hat{c}}{\partial \sigma}+\Delta x \frac{\partial \hat{c}}{\partial \Delta x}
$$

The general discrete expressions for $\hat{c}, \hat{s}$, and $\hat{c}_{\mathrm{g}}$ will depend on $\alpha$ and $\beta$. The MacLaurin series of these dispersion parameters, for the positive direction propagation mode, $G_{1}$, are

$$
\begin{aligned}
\hat{c}= & 1-\frac{1}{6} \sigma^{2}+\frac{1}{24} \sigma^{4}+\frac{1}{4} C r\left(\beta-\frac{C r}{3}\right) \Delta x^{2}+\frac{1}{12}\left(\frac{C r^{2}}{2}-\beta C r-\frac{1}{6}\right) \Delta x^{2} \sigma^{2} \\
& +\frac{1}{16}\left[\beta^{2} C r^{2}+\beta C r\left(\frac{1}{3}-C r^{2}\right)+\frac{C r^{4}}{5}-\frac{4}{45}\right] \Delta x^{4}+\mathcal{O}\left(\sigma^{6}, \Delta x^{6}, \Delta x^{4} \sigma^{2}, \Delta x^{2} \sigma^{4}\right) \\
\hat{c}_{\mathrm{g}}= & 1-\frac{1}{2} \sigma^{2}+\frac{5}{24} \sigma^{4}+\frac{3}{4} C r\left(\beta-\frac{C r}{3}\right) \Delta x^{2}+\frac{5}{12}\left(\frac{C r^{2}}{2}-\beta C r-\frac{1}{6}\right) \Delta x^{2} \sigma^{2} \\
& +\frac{5}{16}\left[\beta^{2} C r^{2}+\beta C r\left(\frac{1}{3}-C r^{2}\right)+\frac{C r^{4}}{5}-\frac{5}{45}\right] \Delta x^{4}+\mathcal{O}\left(\sigma^{6}, \Delta x^{6}, \Delta x^{4} \sigma^{2}, \Delta x^{2} \sigma^{4}\right)
\end{aligned}
$$


and

$$
\hat{s}=\frac{1}{24} \alpha(1-3 \beta C r) \Delta x^{3}+\mathcal{O}\left(\Delta x^{5}, \Delta x^{3} \sigma^{2}\right)
$$

These will be compared to their analytical counterparts. For this purpose, we notice that the MacLaurin series expansion of expressions (7) are

$$
c=1-\frac{1}{6} \sigma^{2}+\frac{1}{24} \sigma^{4}+\mathcal{O}\left(\sigma^{6}\right)
$$

and

$$
c_{\mathrm{g}}=1-\frac{1}{2} \sigma^{2}+\frac{5}{24} \sigma^{4}+\mathcal{O}\left(\sigma^{6}\right)
$$

while the analytical damping ratio is null, i.e. $s=0$.

After setting the $\alpha$ and $\beta$ coefficients to the previously proposed values (see Section 4.1):

$$
\beta=\frac{C r}{3} \quad \text { and } \quad \alpha=\frac{C r^{3}}{1-C r^{2}}
$$

we obtain

$$
\begin{aligned}
\hat{c}= & 1-\frac{1}{6} \sigma^{2}+\frac{1}{24} \sigma^{4}-\frac{1}{72}\left(1-C r^{2}\right) \Delta x^{2} \sigma^{2}-\frac{1}{720}\left(C r^{4}-5 C r^{2}+4\right) \Delta x^{4} \\
& +\mathcal{O}\left(\sigma^{6}, \Delta x^{6}, \Delta x^{4} \sigma^{2}, \Delta x^{2} \sigma^{4}\right) \\
\hat{c}_{\mathrm{g}}= & 1-\frac{1}{2} \sigma^{2}+\frac{5}{24} \sigma^{4}-\frac{5}{72}\left(1-C r^{2}\right) \Delta x^{2} \sigma^{2}-\frac{1}{144}\left(C r^{4}-5 C r^{2}+4\right) \Delta x^{4} \\
& +\mathcal{O}\left(\sigma^{6}, \Delta x^{6}, \Delta x^{4} \sigma^{2}, \Delta x^{2} \sigma^{4}\right)
\end{aligned}
$$

and

$$
\hat{s}=\frac{1}{24} C r^{3} \Delta x^{3}+\mathcal{O}\left(\Delta x^{5}, \Delta x^{3} \sigma^{2}\right)
$$

If we define $\theta=\sigma / \Delta x^{\prime}=h / \Delta x$ as a discretization parameter, we might see that

$$
\begin{aligned}
& \frac{\hat{c}}{c}=1-\frac{1}{36}\left(\frac{1}{20} C r^{4}-\frac{1}{2} C r^{2} \theta^{2}-\frac{1}{4} C r^{2}+\frac{1}{2} \theta^{2}+\frac{1}{5}\right) \Delta x^{4}+\mathcal{O}\left(\Delta x^{6}\right) \\
& \frac{\hat{c}_{\mathrm{g}}}{c_{\mathrm{g}}}=1-\frac{5}{36}\left(\frac{1}{20} C r^{4}-\frac{1}{2} C r^{2} \theta^{2}-\frac{1}{4} C r^{2}+\frac{1}{2} \theta^{2}+\frac{1}{5}\right) \Delta x^{4}+\mathcal{O}\left(\Delta x^{6}\right)
\end{aligned}
$$

This shows us that the leading order truncation terms are fourth order in $\Delta x$ for both the phase velocity and the group velocity, although five times larger for the latter. Both the amplitude and the phase accuracy depend on the wave number and on the depth, as $\Delta x^{\prime}=k \Delta x$ and $\theta=\sigma / \Delta x^{\prime}=h / \Delta x$.

For a coarse mesh, or near the shallow water limit, $\theta \rightarrow 0$, and for a relatively high Courant number, below 1 , the phase velocity might become negative for the first propagation mode, while being null for the second propagation mode (see Figure 4 for $\theta=2$ ). As $\left|G_{2}\right|=1$ for $k \Delta x=\pi$, this might result in a non-damped and non-propagating wave for the shortest resolved wavelength, $\lambda=2 \Delta x$, which will induce the production of numerical noise. Close observation of the real and imaginary parts of $G_{1}$ and $G_{2}$ (see Figure 5) shows us that non-negative phase velocities for the first mode can be enforced provided that the real part of $G_{1}$ is positive or null for $k \Delta x=\pi$. This 

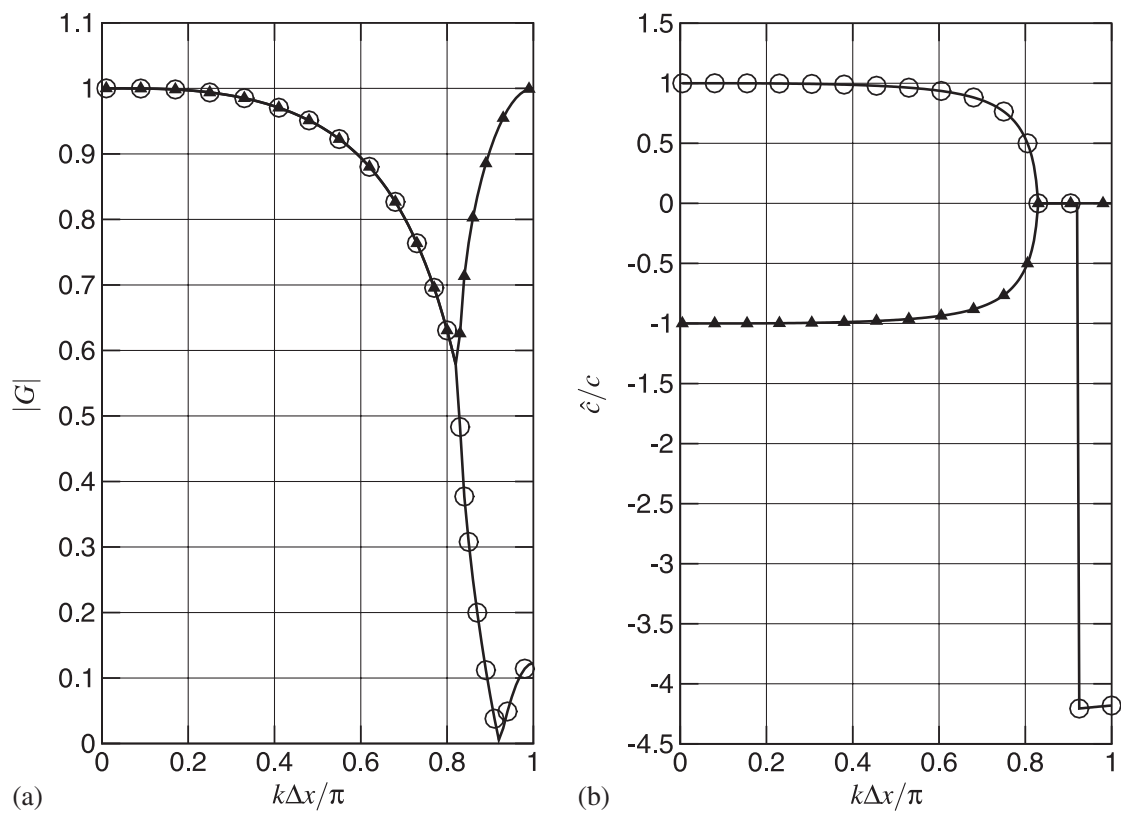

Figure 4. Numerical dissipation and phase velocity error at $\theta=2$ and $C r=0.9$ for the first $(\bigcirc)$ and the second $(\boldsymbol{\Lambda})$ propagation modes: (a) amplification factor and (b) ratio between analytical and numerical phase velocity.

results in the following stability condition:

$$
C r \leqslant \frac{1}{5} \sqrt{-5-10 \theta^{2}+5 \sqrt{6+24 \theta^{2}+4 \theta^{4}}}
$$

This is not a very restrictive condition. Indeed in the shallow water limit, $\theta \rightarrow 0, C r<0.538$, and for $\theta=2$, a usual minimal refinement in dispersive wave modelling, $\mathrm{Cr}$ can reach values up to 0.881 (cf. Figure 6).

We investigated the amplitude and phase accuracy of the scheme for three meshes: a very coarse mesh, $\theta=2$, for which $C r_{\max }=0.881$; a regular mesh, $\theta=5$, for which $C r_{\max }=0.972$; and a refined mesh, $\theta=10$, for which $C r_{\max }=0.992$. From the analysis of Figures 7-9, a first conclusion can be drawn: the choice of a time step will always be a balance between phase and amplitude accuracy. A higher Courant number will induce a higher phase accuracy, but also a larger amplitude error, as it will increase the amplitude dissipation. A detailed analysis will now be worked out for each of the referred discretizations.

For the very coarse mesh, $\theta=2$, the range of validity of the Boussinesq model extends up to $k \Delta x=\pi / 4(k h=\pi / 2)$, and waves are well resolved up to $k \Delta x=\pi / 2(k h=\pi)$. We might expect such a low mesh resolution not to be found on any operational simulation but for a very shallow water, where a small depth will induce a small wave celerity and thus a low Courant number. For the waves in the Boussinesq range of validity, the amplitude error will stay below $0.5 \%$ for $\mathrm{Cr}<0.8$ (cf. Figure 7(a)). Shorter waves, those in the well-resolved range of wave numbers, suffer a amplitude reduction below $1 \%$ for $\mathrm{Cr}<0.6$, and below $3.5 \%$ for $0.6<C r<0.8$. Higher wave 


\section{P. AVILEZ-VALENTE AND F. J. SEABRA-SANTOS}

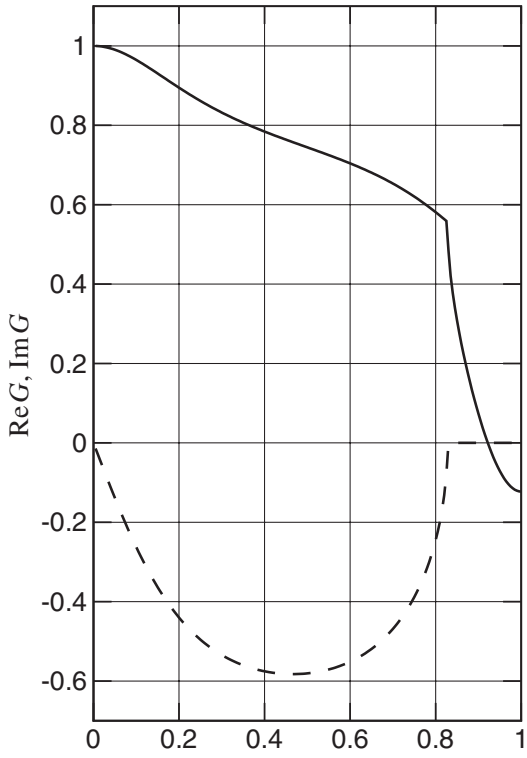

(a)

$k \Delta x / \pi$

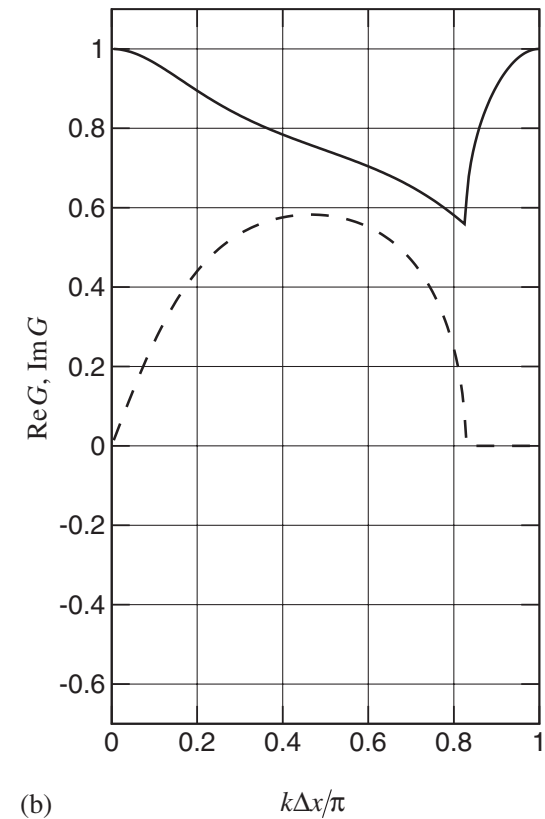

Figure 5. Real (-) and imaginary (- - ) parts of the amplification factor at $\theta=2$ and $C r=0.9$ : (a) first mode, $G_{1}$, and (b) second mode, $G_{2}$.

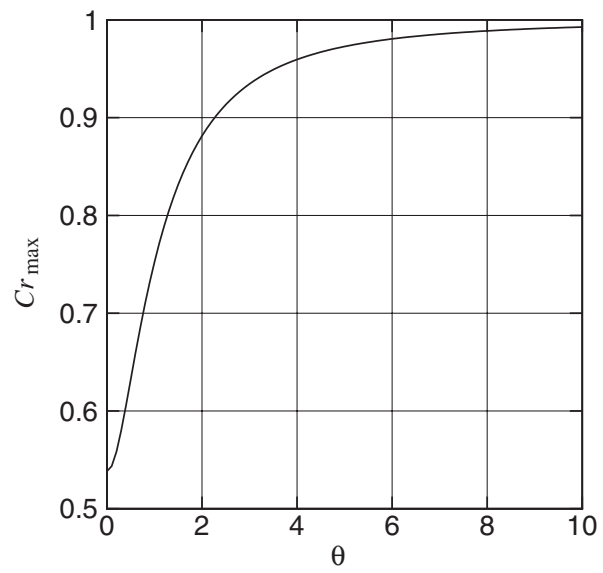

Figure 6. Relationship between maximum Courant number and spatial mesh size $\theta=h / \Delta x$.

numbers will be quickly dissipated. Phase accuracy is somehow worse: the phase error stays below $1 \%$ for $C r>0.8$, and between 1 and $2 \%$ for $0.4<C r<0.8$, in the model range of validity; for the shorter but well-resolved waves, the phase error will attain a maximum between 5 and $10 \%$ for $0.4<C r<0.8$ (cf. Figure 7(b)). 

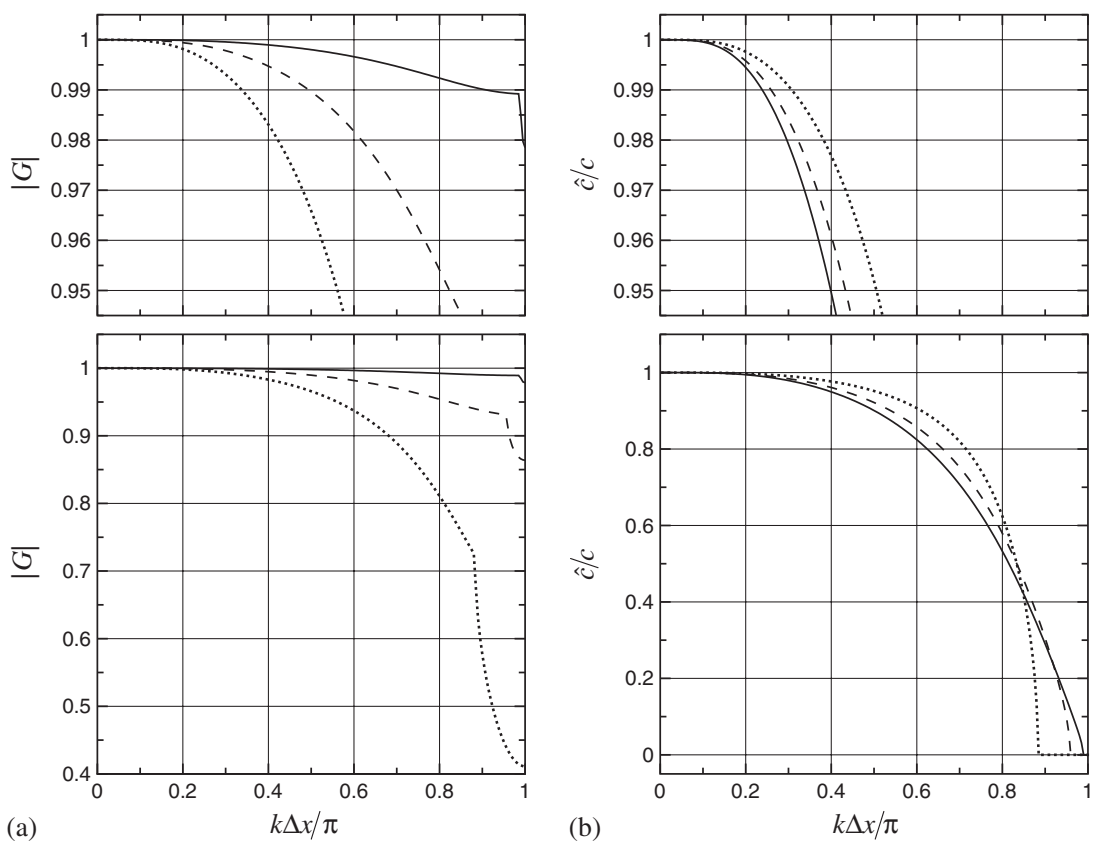

Figure 7. Numerical dissipation and phase velocity error at $\theta=2$ and $C r=0.4(-), C r=0.6(--)$, and $C r=0.8(\cdots)$ : (a) amplification factor and (b) ratio between analytical and numerical phase velocity.

For the regular mesh, $\theta=5$, the range of validity of the Boussinesq model extends up to $k \Delta x=\pi / 10$, and well-resolved waves will reach $k \Delta x=\pi / 5$. One can notice (see Figure $8(\mathrm{a})$ ) that even for $C r=0.9$, the amplitude error will stay well below $0.5 \%$ up to the well-resolved waves limit. For $C r=0.9$, only the very short waves will suffer a dissipation above $5 \%$, attaining $35 \%$ for the shortest mesh resolved wave. This shows the highly selective character of the dissipation mechanism.

For the refined mesh, $\theta=10$, the range of validity of the Boussinesq model is $k \Delta x=\pi / 20$, and the wave well-resolved limit is $k \Delta x=\pi / 10$. For the Boussinesq range of validity, the amplitude error stays well below $0.005 \%$ for all Courant numbers. The maximum $0.005 \%$ dissipation error is attained for $\mathrm{Cr}=0.9$ in the well-resolved waves limit. But even for this refined mesh, the stabilizing mechanism still induces some dissipation on the non-propagating numerical waves attaining a $10 \%$ dissipation for $C r=0.9$ and $k \Delta x=\pi(\lambda=2 \Delta x)$. For $C r=0.7$ this limiting dissipation is reduced to ca. $1.5 \%$ (cf. Figure 9(a)). On what concerns the phase error, this is an order of magnitude larger than the amplitude error. Nevertheless, the phase error stays below $0.05 \%$ for all waves within the model validity range and below $0.25 \%$ for all waves within the well-resolved range, with a minimum of $0.05 \%$ for $C r=0.9$ (cf. Figure 9(b)).

\subsection{Predictor-corrector}

A von Neumann stability analysis is performed for the predictor-corrector scheme to be used for integration within a time-slab. The value for $G$ at each corrector step depends on the adopted 

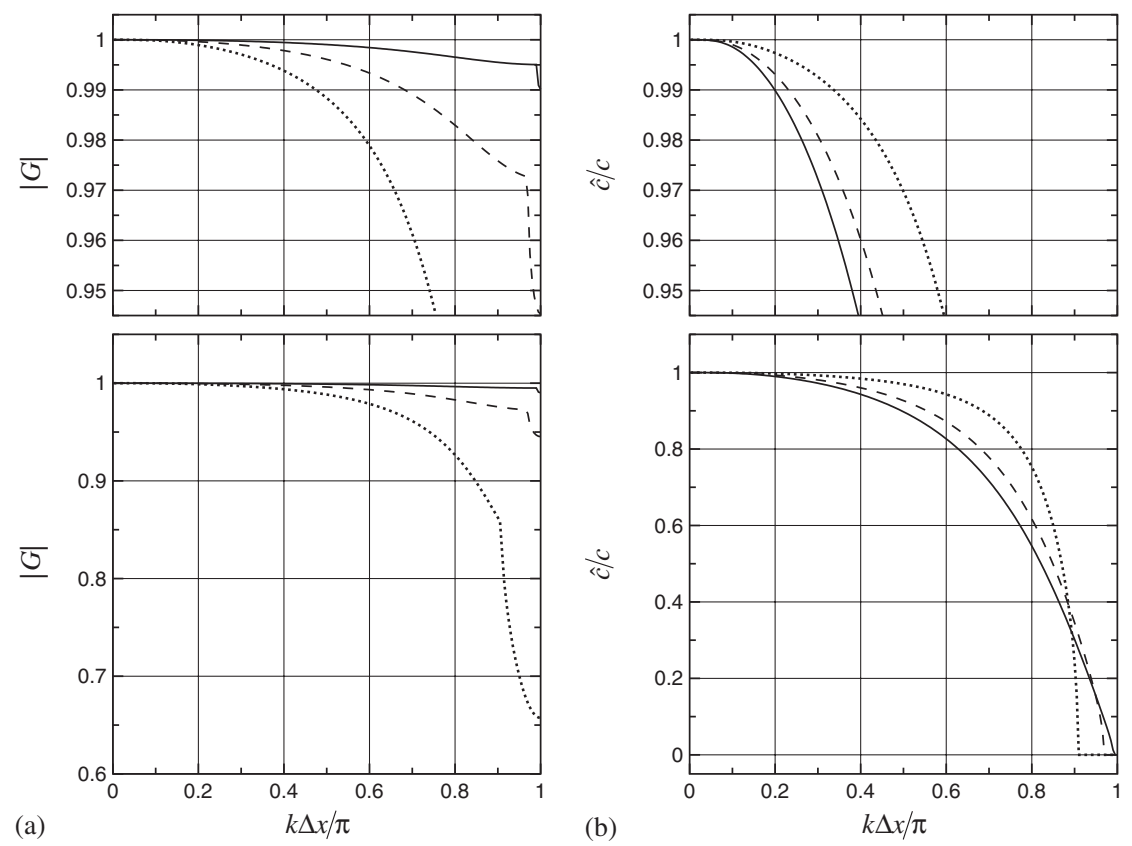

Figure 8. Numerical dissipation and phase velocity error at $\theta=5$ and $C r=0.5$ (-), $C r=0.7$ (- - , and $C r=0.9(\cdots)$ : (a) amplification factor and (b) ratio between analytical and numerical phase velocity.

expressions for $\alpha$ and $\beta$, i.e. $\alpha_{k}$ and $\beta_{k}$, in the predictor step and in the previous corrector step. We analyse the MacLaurin series expansions of $\hat{c}$ and $\hat{s}$ at each inner iteration step.

Predictor step: For this step, the MacLaurin expansions of the numerical phase velocity and damping ratio are

$$
\begin{gathered}
\hat{c}_{0}=1-\frac{1}{6} \sigma^{2}-\left(\frac{1}{3} C r^{2}-\frac{1}{2} \alpha_{0} C r+\frac{1}{8} \alpha_{0}^{2}\right) \Delta x^{2}+\mathcal{O}\left(\Delta x^{4}, \Delta x^{2} \sigma^{2}, \sigma^{4}\right) \\
\hat{s}_{0}=\frac{1}{2}\left(\alpha_{0}-C r\right) \Delta x+\left(\frac{1}{24} \alpha_{0}+\frac{1}{4} C r \alpha_{0}^{2}-\frac{1}{2} C r^{2} \alpha_{0}+\frac{1}{4} C r^{3}\right) \Delta x^{3} \\
-\frac{1}{6}\left(\alpha_{0}-C r\right) \Delta x \sigma^{2}+\mathcal{O}\left(\Delta x^{5}, \Delta x^{3} \sigma^{2}\right)
\end{gathered}
$$

Choosing

$$
\alpha_{0}=C r
$$

optimizes the damping ratio in the predictor step making it third order, while a second-order dispersion error stills exists. It is obvious from Equation (27) that the coefficient $\beta$ does not play any role on this step, and therefore $\beta_{0}=0$. The phase velocity and the damping ratio are now such that

$$
\begin{gathered}
\hat{c}_{0}=1-\frac{1}{6} \sigma^{2}+\frac{1}{24} C r^{2} \Delta x^{2}+\mathcal{O}\left(\Delta x^{4}, \Delta x^{2} \sigma^{2}, \sigma^{4}\right) \\
\hat{s}_{0}=\frac{1}{24} \Delta x^{3}+\mathcal{O}\left(\Delta x^{5}, \Delta x^{3} \sigma^{2}\right)
\end{gathered}
$$


A PETROV-GALERKIN FEM FOR THE BOUSSINESQ WAVE MODEL
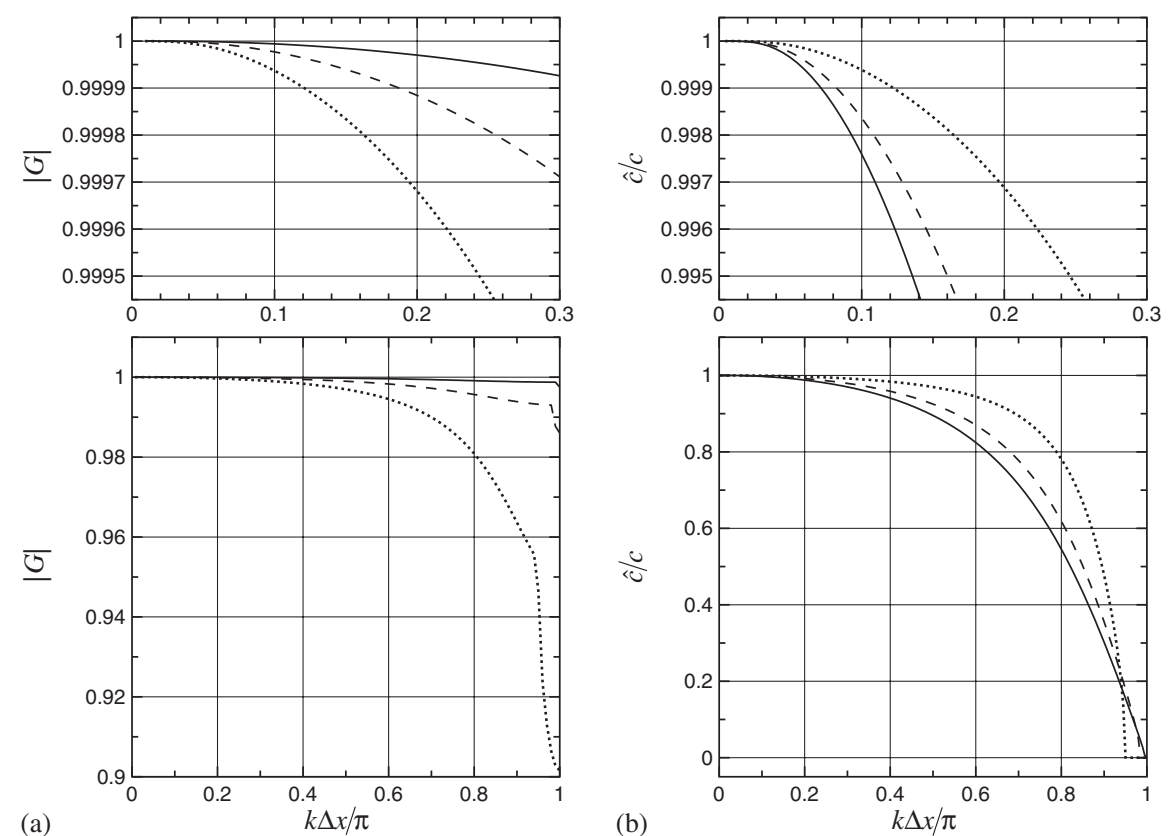

Figure 9. Numerical dissipation and phase velocity error at $\theta=10$ and $C r=0.5(-), C r=0.7$ (- -), and $C r=0.9(\cdots)$ : (a) amplification factor and (b) ratio between analytical and numerical phase velocity.

To avoid negative phase velocities for the first mode of propagation, we impose that $\operatorname{Re} G_{1} \geqslant 0$ for $k \Delta x=\pi$, from where a new stability condition arises:

$$
C r \leqslant \frac{1}{6} \sqrt{3+12 \theta^{2}}
$$

This is a much less restrictive condition than $(51)$, except for $\theta<\sqrt{1955} / 34 \approx 1.3$. As in actual computations $\theta \geqslant 2$, condition (51) still applies.

First corrector step: In this step, both the $\alpha_{1}$ and $\beta_{1}$ coefficients play an active role. The MacLaurin expansions for the wave velocity and damping ratio are now

$$
\begin{gathered}
\hat{c}_{1}=1-\frac{1}{6} \sigma^{2}+\frac{C r}{4}\left(\beta_{1}-\frac{C r}{3}\right) \Delta x^{2}+\mathcal{O}\left(\Delta x^{4}, \Delta x^{2} \sigma^{2}, \sigma^{4}\right) \\
\hat{s}_{1}=\frac{1}{8}\left[\left(\frac{1}{3}-\frac{C r^{2}}{2}\right) \alpha_{1}-C r^{2}\left(\beta_{1}-\frac{C r}{2}\right)\right] \Delta x^{3}+\mathcal{O}\left(\Delta x^{5}, \Delta x^{3} \sigma^{2}\right)
\end{gathered}
$$

It is easy to see that the choice of

$$
\beta_{1}=\frac{C r}{3}
$$




\section{P. AVILEZ-VALENTE AND F. J. SEABRA-SANTOS}

will lead to a fourth-order approximation for the phase velocity. On the other hand, the dissipation term is not yet similar to the one in (46c). Hence, we preferred to set

$$
\alpha_{1}=0
$$

which still keeps a third-order dissipative term in the first corrector step. The resulting MacLaurin expansions are now

$$
\begin{gathered}
\hat{c}_{1}=1-\frac{1}{6} \sigma^{2}+\frac{1}{24} \sigma^{4}-\frac{1}{72}\left(1-C r^{2}\right) \sigma^{2} \Delta x^{2}+\frac{1}{1440}\left(3 C r^{4}-8 C r^{2}-20\right) \Delta x^{4} \\
+\mathcal{O}\left(\Delta x^{6}, \Delta x^{4} \sigma^{2}, \Delta x^{2} \sigma^{4}, \sigma^{6}\right) \\
\hat{s}_{1}=\frac{1}{48} C r^{3} \Delta x^{3}+\mathcal{O}\left(\Delta x^{5}, \Delta x^{3} \sigma^{2}\right)
\end{gathered}
$$

The leading dissipative term is positive for any value of the Courant number. In order to avoid non-positive phase velocities for the first mode of propagation, the stability condition for the first predictor step is

$$
C r \leqslant \frac{1}{12}\left(1728+6912 \theta^{2}\right)^{1 / 4}
$$

which is less restrictive than (51) for all $\theta$, but $\theta<\sqrt{3} / 6 \approx 0.288$. Thus, for operational conditions, $\theta \geqslant 2$, condition (51) still applies.

Second corrector step: At this step, the MacLaurin expansions of the linear phase velocity and damping ratio are

$$
\begin{gathered}
\hat{c}_{2}=1-\frac{1}{6} \sigma^{2}+\frac{C r}{4}\left(\beta_{2}-\frac{C r}{3}\right) \Delta x^{2}+\mathcal{O}\left(\Delta x^{4}, \Delta x^{2} \sigma^{2}, \sigma^{4}\right) \\
\hat{s}_{2}=\frac{1}{24}\left[\left(1-C r^{2}\right) \alpha_{2}-3 C r^{2}\left(\beta_{2}-\frac{C r}{3}\right)\right] \Delta x^{3}+\mathcal{O}\left(\Delta x^{5}, \Delta x^{3} \sigma^{2}\right)
\end{gathered}
$$

Comparing these results with those found in Section 4.1 shows that the second corrector step already achieves the same accuracy of the equivalent differential equations, for similar $\alpha$ and $\beta$ coefficients:

$$
\beta_{2}=\frac{C r}{3} \quad \text { and } \quad \alpha_{2}=\frac{C r^{3}}{1-C r^{2}}
$$

Therefore, one finally obtains

$$
\begin{gathered}
\hat{c}_{2}=1-\frac{1}{6} \sigma^{2}+\frac{1}{24} \sigma^{4}-\frac{1}{72}\left(1-C r^{2}\right) \sigma^{2} \Delta x^{2}-\frac{1}{1440} \frac{28 C r^{6}+5 C r^{4}-34 C r^{2}+8}{1-C r^{2}} \Delta x^{4} \\
+\mathcal{O}\left(\Delta x^{6}, \Delta x^{4} \sigma^{2}, \Delta x^{2} \sigma^{4}, \sigma^{6}\right) \\
\hat{s}_{2}=\frac{1}{24} C r^{3} \Delta x^{3}+\mathcal{O}\left(\Delta x^{5}, \Delta x^{3} \sigma^{2}\right)
\end{gathered}
$$

To avoid non-positive phase velocities for the first mode of propagation combined with zero phase velocities for the second propagation mode, the stability condition for the second predictor step is

$$
C r \leqslant \frac{1}{12}\left(248832+995328 \theta^{2}\right)^{1 / 6}
$$

which is less restrictive than (51) for all $\theta$, and hence (51) still applies. 
A PETROV-GALERKIN FEM FOR THE BOUSSINESQ WAVE MODEL
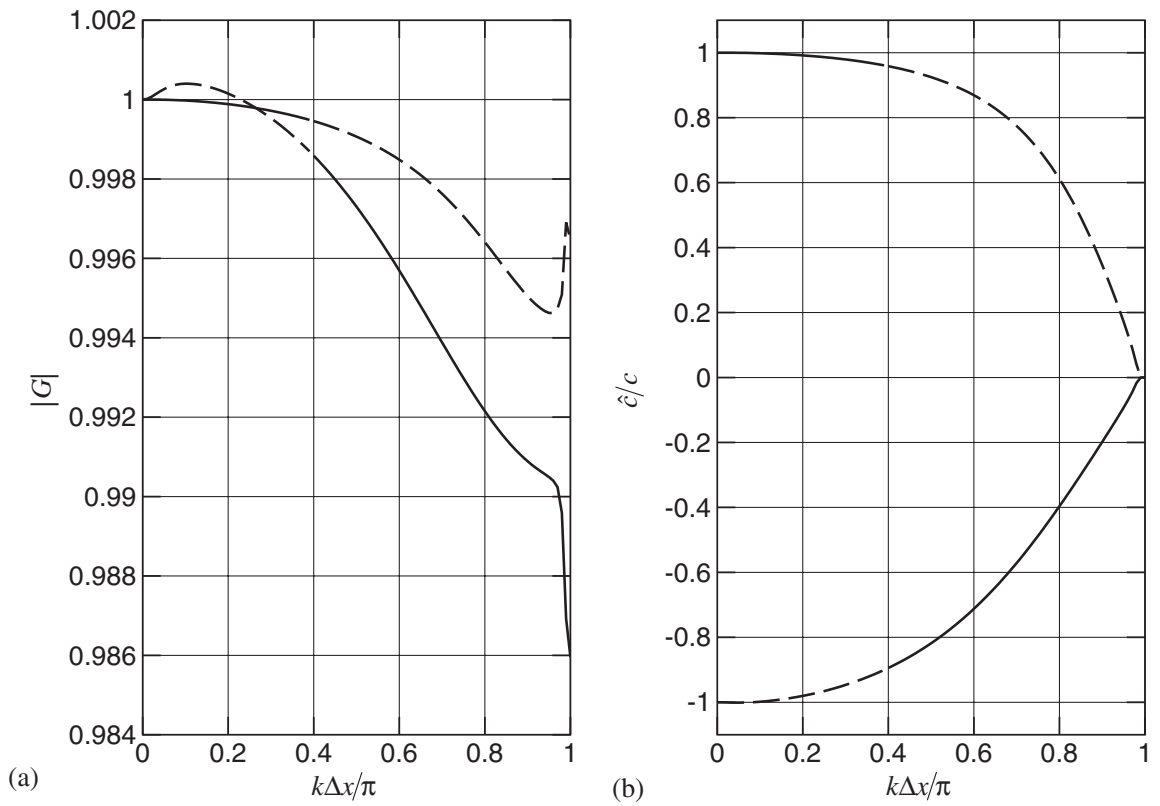

Figure 10. Numerical dissipation and phase velocity error for the second corrector step, at $\theta=10$ and $C r=0.7$. First propagation mode (-) and second propagation mode (- -): (a) amplification factor and (b) ratio between analytical and numerical phase velocity.

The behaviour of the higher-order terms in the predictor-corrector scheme is somehow different from the behaviour expected from the linear analysis conducted in Section 4.2. In the predictorcorrector scheme, a change in signal always occurs for the phase velocity due to a change in signal in the imaginary parts of the propagation factors $G_{1}$ and $G_{2}$. Nevertheless, the fact that this change occurs simultaneously on both modes guarantees the continuity of both the phase velocity and the amplification factor (see Figure 10). For large values of $\mathrm{Cr}$ (below 1), the first propagation mode might even become negative for all values of $k \Delta x$. Analysis of the combined $G_{1}-G_{2}$ positive propagating modes shows us that on the second corrector step the phase velocity is null only for $k \Delta x=\pi$ (cf. Figure 11). This is somehow an improvement over the behaviour of the numerical scheme as predicted in Section 4.2.

Third corrector step: The MAPLE ${ }^{\mathrm{TM}}$ software was used for the symbolic computations, and it was impossible to get results for a third corrector step as the memory allocation failed. Nevertheless, in an earlier work [43] the authors showed that a similar scheme for the RLW equation would display the same leading error terms for both the second and the third corrector steps. The RLW model is the Boussinesq model counterpart for kinematic waves, and therefore one might expect that a similar behaviour will be found within the framework of the latter model. Besides that, comparison with the non-linear analysis in Section 4.1 allows us to conclude that the $\alpha$ and $\beta$ coefficients correction terms have already reached their final form and that further corrector steps shall use the same coefficients. Thus, a third corrector step with

$$
\beta_{3}=\beta_{2}=\frac{C r}{3} \quad \text { and } \quad \alpha_{3}=\alpha_{2}=\frac{C r^{3}}{1-C r^{2}}
$$



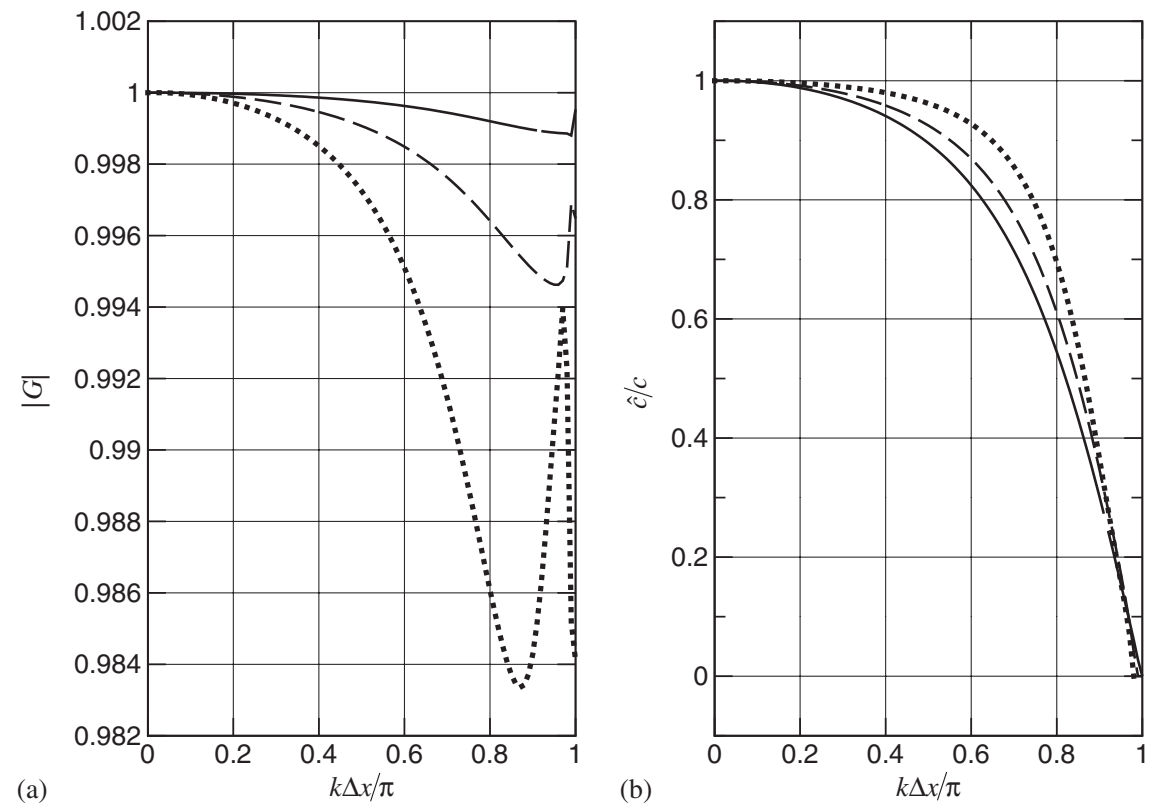

Figure 11. Numerical dissipation and phase velocity error for the second corrector step, at $\theta=10$ and $C r=0.5(-), C r=0.7(--)$, and $C r=0.9(\cdots)$ : (a) amplification factor and

(b) ratio between analytical and numerical phase velocity.

shall keep the accuracy and stability of the scheme, while enhancing the precision of the non-linear terms. Although further analysis is still necessary, the results obtained herein for this formulation (see Section 5) seem to confirm this assumption.

\section{NUMERICAL EXPERIMENTS AND CONVERGENCE RESULTS}

Four numerical experiments are presented: the propagation of a solitary wave, the oscillation of a flat bottom closed basin, the oscillation of a non-flat bottom closed basin, and the propagation of a periodic wave over a submerged bar. Mass conservation of the numerical scheme is analysed, along with some accuracy and convergence properties. Unless stated otherwise, one predictor + two corrector steps were used for inner iteration within each time step.

\subsection{Propagation of a solitary wave}

For a flat bottom, the Boussinesq model (2) has a solitary wave solution [44] with a closed form given by [45]

$$
\begin{gathered}
\zeta=\frac{a \operatorname{sech}^{2}\left[\kappa\left(x-c t-x_{0}\right)\right]}{1+a \tanh ^{2}\left[\kappa\left(x-c t-x_{0}\right)\right]} \\
\bar{u}=c \frac{\zeta}{h_{0}+\zeta}
\end{gathered}
$$


with

$$
\kappa=\sqrt{\frac{3}{4} \frac{a}{h_{0}^{2}} \frac{1}{\left(h_{0}+0.68 a\right)}}
$$

and

$$
c=\sqrt{g h_{0}} \sqrt{\frac{6\left(h_{0}+a\right)^{2}}{a^{2}\left(3 h_{0}+2 a\right)}\left[\left(h_{0}+a\right) \ln \left(1+\frac{a}{h_{0}}\right)-a\right]}
$$

where $a$ is the wave amplitude, $h_{0}$ is the water depth at rest, $c$ is the wave velocity, and $\kappa$ is the outskirt decay parameter.

The computational domain is long enough so that both $\zeta$ and $\bar{u}$ vanish at the boundaries throughout the entire numerical simulation: $x \in[-50,1050] \mathrm{m}$. The water depth at rest is $h_{0}=1.0 \mathrm{~m}$ and $g=9.81 \mathrm{~m} / \mathrm{s}^{2}$. The finite element mesh size is $\Delta x=0.1 \mathrm{~m}$, hence $\theta=10$, and $\Delta t=0.025 \mathrm{~s}$. The Courant number is $C r=\sqrt{g h_{0}} \Delta t / \Delta x=0.783$. Two solitary waves, a weakly non-linear one, and a strongly non-linear one will be simulated.

The $L_{2}$ - and $L_{\infty}$-norms,

$$
\begin{gathered}
L_{2}=\frac{\left[\int_{0}^{L}\left(\hat{\zeta}^{(n)}-\zeta^{(\text {exact })}\right)^{2} \mathrm{~d} x\right]^{1 / 2}}{\int_{0}^{L} \zeta^{(\text {exact })} \mathrm{d} x} \approx \frac{\left[\sum_{1}^{N}\left(\hat{\zeta}_{j}^{(n)}-\zeta_{j}^{\text {(exact) }}\right)^{2}\right]^{1 / 2}}{\sum_{1}^{N} \zeta_{j}^{\text {(exact) }}} \\
L_{\infty}=\frac{\max \left|\hat{\zeta}^{(n)}-\zeta^{(\text {exact })}\right|}{\max \left|\zeta^{(\text {exact })}\right|} \approx \frac{\max _{j}\left|\hat{\zeta}_{j}^{(n)}-\zeta_{j}^{(\text {exact })}\right|}{\max _{j}\left|\zeta_{j}^{\text {(exact) }}\right|}
\end{gathered}
$$

measuring the mean and maximum differences between the analytical and numerical solution, will show how well the numerical scheme predicts the amplitude and position of the solitary wave along its propagation.

For the weakly non-linear wave, $a=0.1 \mathrm{~m}$, hence $\varepsilon=0.1$, one has that $c=3.282 \mathrm{~m} / \mathrm{s}$ and $\kappa=$ $0.2650 \mathrm{~m}^{-1}$. A characteristic wavelength is defined as $\lambda=2 \pi / \kappa$ and calculated as $23.71 \mathrm{~m}$, thus $\lambda / \Delta x=237$, and the corresponding wave period, $T=\lambda / c$, is estimated as $7.223 \mathrm{~s}$. The initial wave crest is located at $x_{0}=0$. The numerical experiment is run up to $t=300 \mathrm{~s}$, i.e. over 40 wave periods. An excellent match is found between the numerical results and the closed-form solution (cf. Figure 12(a)). Close observation of the wave properties (see Table I) shows that the total amplitude decrease stays below $0.05 \%$, while the wave velocity is reproduced with an error below $0.5 \times 10^{-4} \mathrm{~m} / \mathrm{s}$. Mass is conserved throughout the simulation. This is consistent with the introduced stability mechanism showing that stability can be achieved without any mass loss. The evolution of the $L_{2}$ - and $L_{\infty}$-error norms, which display a monotonic increase at a very slow rate, demonstrate that the scheme is both stable and accurate, providing a good capture of both shape and position of the disturbance.

For the strongly non-linear wave, $a=0.6 \mathrm{~m}$, hence $\varepsilon=0.6$, one has that $c=3.892 \mathrm{~m} / \mathrm{s}$ and $\kappa=0.5653 \mathrm{~m}^{-1}$. The estimated wavelength is $\lambda=11.11 \mathrm{~m}$, thus $\lambda / \Delta x=111$, and the wave period is $T=2.8556 \mathrm{~s}$. The wave crest is located at $x_{0}=0$ for $t=0$, and the numerical experiment is run 


\section{P. AVILEZ-VALENTE AND F. J. SEABRA-SANTOS}
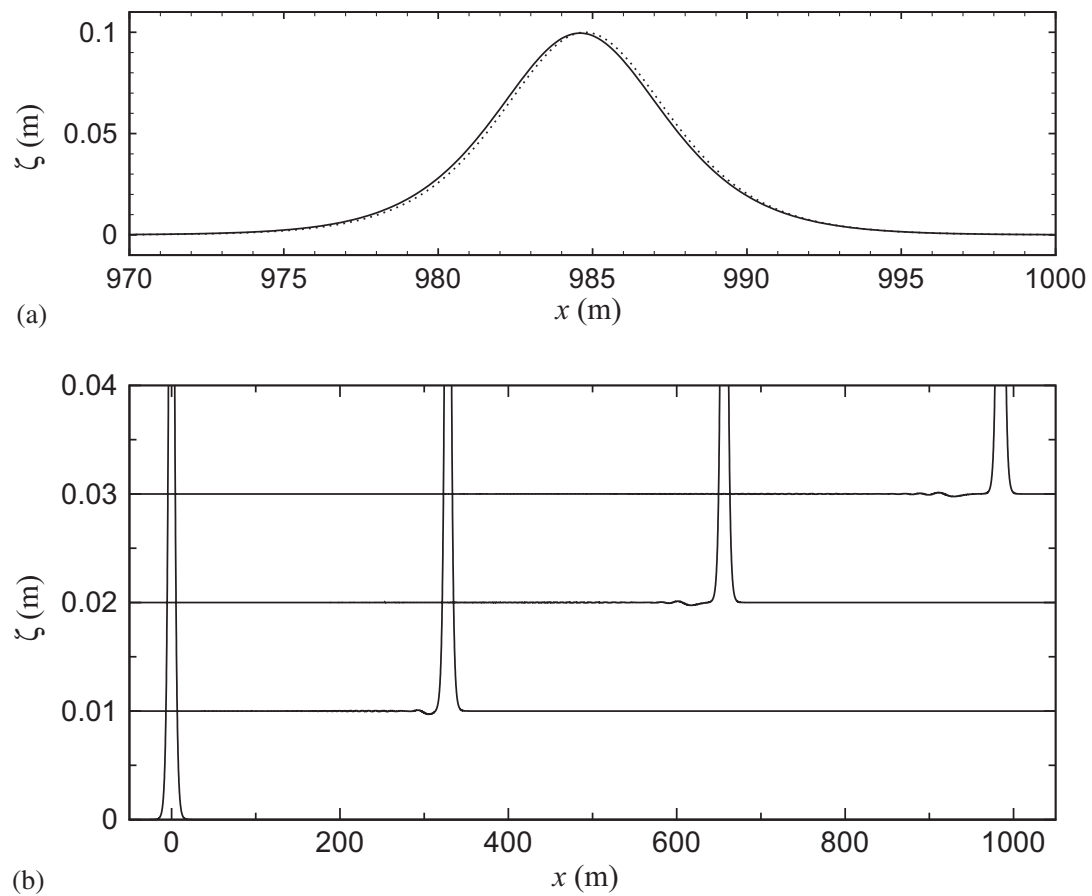

Figure 12. Weakly non-linear solitary wave, $\varepsilon=0.1$, propagating over 40 wavelengths: (a) numerical solution $(--)$ and closed form ( $\ldots)$ for $t=300 \mathrm{~s}$ and (b) dispersive tails at $t=0,100,200$, and 300s. Results for $t=100,200$, and $300 \mathrm{~s}$ are shifted with $0.01,0.02$, and $0.03 \mathrm{~m}$, respectively, relative to the vertical axis.

up to $t=150$ s, i.e. over 50 wave periods. Two runs were executed: the first run with one predictor + two corrector steps; the second run with one predictor + three corrector steps.

For the first run, the wave amplitude is underpredicted, and a phase error is evident (cf. Figure 13(a)). Nevertheless, the amplitude decrease is only ca. $2.1 \%$ after 50 wave periods (see Table I). The phase error is a result of the small decrease in the wave velocity, induced by the diminishing wave amplitude. In Table I one can also see that mass is conserved throughout the experiment, while the $L_{2}$ - and $L_{\infty}$-error norms increase at a high rate, reaching 0.26 and 0.46 at $t=150 \mathrm{~s}$, respectively.

For the second run, the computation registered a small phase error (cf. Figure 13(a)) although the wave velocity seems to be mostly well resolved (cf. Table I) throughout the experiment. The final wave amplitude shows a decrease below $0.6 \%$. Mass is conserved throughout the experiment. The $L_{2}$ - and $L_{\infty}$-error norms, although very small after the $150 \mathrm{~s}$ long simulation, respectively, 0.082 and 0.145 , are an order of magnitude larger than the values registered for the $0.1 \mathrm{~m}$ amplitude wave. This is an expected feature as a non-linear truncation error of $\mathcal{O}\left(\varepsilon \Delta x^{2}\right)$ exists in the formulation (see Section 4.1).

A slower dispersive wave was detected trailing behind the solitary wave for both cases (cf. Figures 12(b) and 13(b)). The constant velocity and amplitude displayed by these dispersive waves seem to indicate that they result from an adjustment of the initial closed-form wave profile to the final numerical wave profile. 
A PETROV-GALERKIN FEM FOR THE BOUSSINESQ WAVE MODEL

Table I. Solitary wave propagation.

\begin{tabular}{|c|c|c|c|c|c|c|}
\hline $\begin{array}{l}t \\
\text { (s) }\end{array}$ & $\begin{array}{c}a \\
(\mathrm{~m})\end{array}$ & $\begin{array}{c}x_{\text {crest }} \\
(\mathrm{m})\end{array}$ & $\begin{array}{l}c_{\text {mean }} \\
(\mathrm{m} / \mathrm{s})\end{array}$ & $\begin{array}{c}\text { Mass } \\
\left(\mathrm{m}^{2}\right)\end{array}$ & $L_{2}$-norm & $L_{\infty}$-norm \\
\hline \multicolumn{7}{|c|}{$\varepsilon=0.1$, One predictor + two correctors } \\
\hline 0 & 0.1 & 0 & - & 0.73097 & 0.0 & 0.0 \\
\hline 50 & 0.09982 & 164.1 & 3.282 & 0.73097 & 0.00181819 & 0.00448528 \\
\hline 100 & 0.09972 & 328.2 & 3.282 & 0.73097 & 0.00303793 & 0.00798591 \\
\hline 150 & 0.09968 & 492.3 & 3.282 & 0.73097 & 0.00392112 & 0.01031842 \\
\hline 200 & 0.09965 & 656.4 & 3.282 & 0.73097 & 0.00468373 & 0.01236748 \\
\hline 250 & 0.09962 & 820.5 & 3.282 & 0.73097 & 0.00542512 & 0.01441408 \\
\hline 300 & 0.09959 & 984.6 & 3.282 & 0.73097 & 0.00618484 & 0.01654256 \\
\hline \multicolumn{7}{|c|}{$\varepsilon=0.6$, One predictor + two correctors } \\
\hline 0 & 0.6 & 0 & - & 1.8060 & 0.0 & 0.0 \\
\hline 25 & 0.5965 & 97.2 & 3.889 & 1.8060 & 0.0220270 & 0.0304642 \\
\hline 50 & 0.5943 & 194.4 & 3.889 & 1.8060 & 0.0448079 & 0.0729648 \\
\hline 75 & 0.5930 & 291.6 & 3.889 & 1.8060 & 0.0813827 & 0.1403265 \\
\hline 100 & 0.5909 & 388.7 & 3.884 & 1.8060 & 0.1304228 & 0.2297555 \\
\hline 125 & 0.5894 & 485.8 & 3.884 & 1.8060 & 0.1900138 & 0.3377902 \\
\hline 150 & 0.5874 & 582.8 & 3.880 & 1.8060 & 0.2571908 & 0.4557935 \\
\hline \multicolumn{7}{|c|}{$\varepsilon=0.6$, One predictor + three correctors } \\
\hline 0 & 0.6 & 0 & - & 1.8060 & 0.0 & 0.0 \\
\hline 25 & 0.5977 & 97.2 & 3.888 & 1.8060 & 0.0176478 & 0.0229320 \\
\hline 50 & 0.5980 & 194.5 & 3.892 & 1.8060 & 0.0248693 & 0.0368565 \\
\hline 75 & 0.5977 & 291.8 & 3.892 & 1.8060 & 0.0350702 & 0.0565730 \\
\hline 100 & 0.5969 & 389.0 & 3.888 & 1.8060 & 0.0481238 & 0.0812710 \\
\hline 125 & 0.5969 & 486.3 & 3.892 & 1.8060 & 0.0638958 & 0.1107875 \\
\hline 150 & 0.5965 & 583.5 & 3.888 & 1.8060 & 0.0822760 & 0.1448000 \\
\hline
\end{tabular}

Convergence: The difference between two numerical solutions is measured by the $L_{1}$-error norm as

$$
L_{1}^{(n)}=\frac{\sum_{j=1}^{N^{(n)}}\left\|\hat{\zeta}_{j}^{(n)}-\hat{\zeta}_{j}^{(n+1)}\right\|}{\sum_{j=1}^{N^{(n)}}\left\|h_{0}+\hat{\zeta}_{j}^{(n+1)}\right\|}
$$

where $\hat{\zeta}^{(n)}$ and $\hat{\zeta}^{(n+1)}$ are the numerical solutions for the $n$th and $(n+1)$ th meshes, respectively, $N^{(n)}$ is the number of points in the $n$th mesh, and $h_{0}$ is the water depth at rest. The Cauchy convergence rate for mesh (or time) refinement can be estimated experimentally from a geometric sequence of $L_{1}$-norms, by successively refining the numerical resolution, i.e.

$$
r=\frac{\log \left[\left(L_{1}^{(n)}-L_{1}^{(n+1)}\right) /\left(L_{1}^{(n+1)}-L_{1}^{(n+2)}\right)\right]}{\log Q}
$$

where

$$
Q=\frac{\Delta x^{(n)}}{\Delta x^{(n+1)}}=\frac{\Delta x^{(n+1)}}{\Delta x^{(n+2)}} \quad \text { or } \quad Q=\frac{\Delta t^{(n)}}{\Delta t^{(n+1)}}=\frac{\Delta t^{(n+1)}}{\Delta t^{(n+2)}}
$$

Computations were carried out for about two wave periods. 


\section{P. AVILEZ-VALENTE AND F. J. SEABRA-SANTOS}
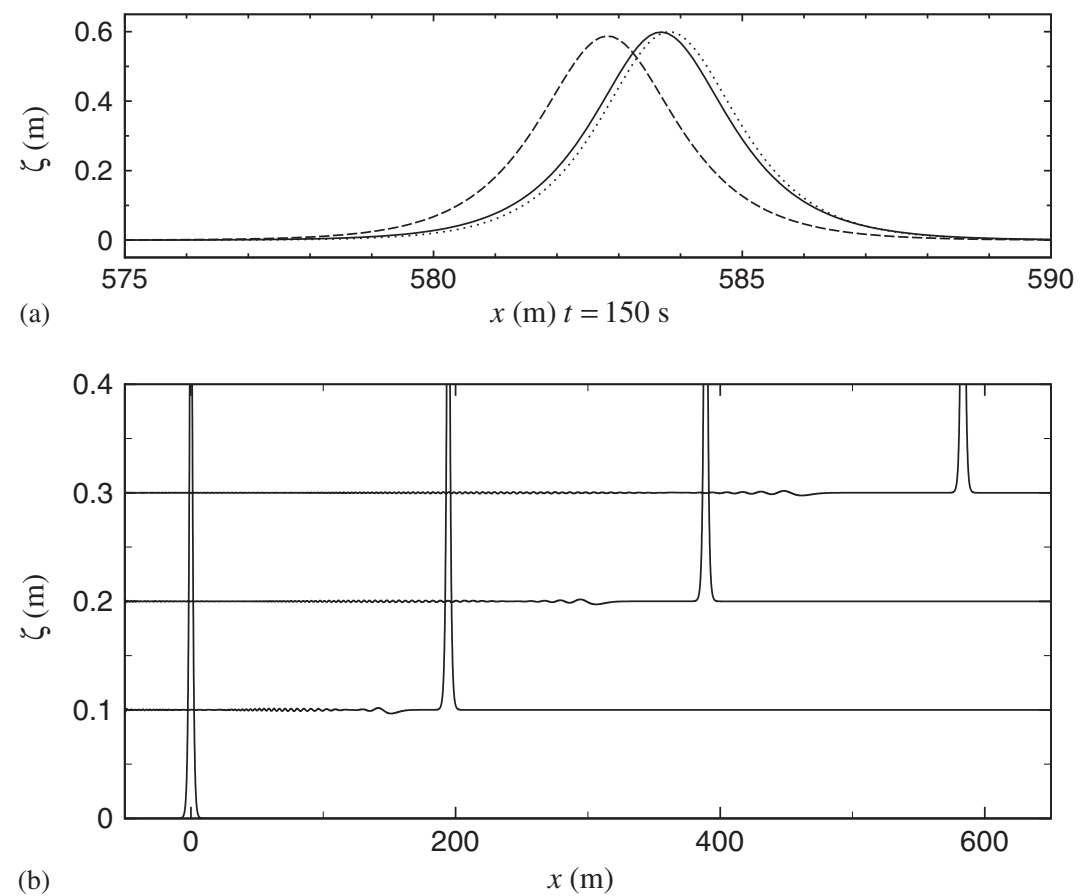

Figure 13. Strongly non-linear solitary wave, $\varepsilon=0.6$, propagating over 50 wavelengths: (a) numerical solution for one predictor + three corrector steps $(-)$; numerical solution for one predictor + two corrector steps $(--)$; closed form $(\cdots)$ and (b) dispersive tails at $t=0,50,100$, and $150 \mathrm{~s}$, for one predictor + three corrector steps. Results for $t=100,200$, and $300 \mathrm{~s}$ are shifted with $0.1,0.2$, and $0.3 \mathrm{~m}$, respectively, relative to the vertical axis.

The spatial discretization convergence was analysed using a fixed time step $\Delta t=0.0075 \mathrm{~s}$ and two geometric sequences for $\Delta x: \Delta x=0.1 / 2^{n-1} \mathrm{~m}, n=1 \ldots 6$, and $\Delta x=0.6667 / 2^{n-1} \mathrm{~m}, n=1 \ldots .5$. Results are presented in Figure 14(a). For the weakly non-linear wave, $\varepsilon=0.1$, the mean convergence rate for spatial discretization is $\bar{r}=2.09$, while for the strongly non-linear wave, $\varepsilon=0.6$, $\bar{r}=2.19$. The ideal slope for a second-order spatial discretization convergence is well recovered, except for the very coarse mesh with $\theta=1$. The results clearly show that the truncation error associated with the spatial discretization is $\mathcal{O}\left(\Delta x^{2}\right)$. As a truncation error term of $\mathcal{O}\left(\varepsilon \Delta x^{2}, \sigma^{2} \Delta x^{2}\right)$ exists, mesh or time-step refinement can only lead to a second-order rate of convergence, as $\varepsilon$ and $\sigma$ remain constant.

A similar convergence test was performed for time discretization. A constant mesh size, $\Delta x=$ $\frac{1}{6} \mathrm{~m}$, hence $\theta=6$, was used, along with two geometric sequences for $\Delta t: \Delta t=0.05 / 2^{n-1} \mathrm{~s}, n=1 \ldots 6$, and $\Delta t=0.03333 / 2^{n-1} \mathrm{~s}, n=1 \ldots 5$. Results are presented in Figure 14(b). Again the second-order ideal slope is well recovered except for the first time-step size, for which $C r=0.94$, which might be too close to the scheme stability limit. The computed mean convergence rates were $\bar{r}=2.81$ and $\bar{r}=2.51$ for $\varepsilon=0.1$ and 0.6 , respectively. These values were corrected to $\bar{r}^{*}=2.30$ and 2.40 after removal of the results for the coarser time-step size, confirming that the truncation error related to the time discretization is $\mathcal{O}\left(\Delta t^{2}\right)$. As expected, a strong non-linearity does not affect the convergence rate of the formulation. 
A PETROV-GALERKIN FEM FOR THE BOUSSINESQ WAVE MODEL
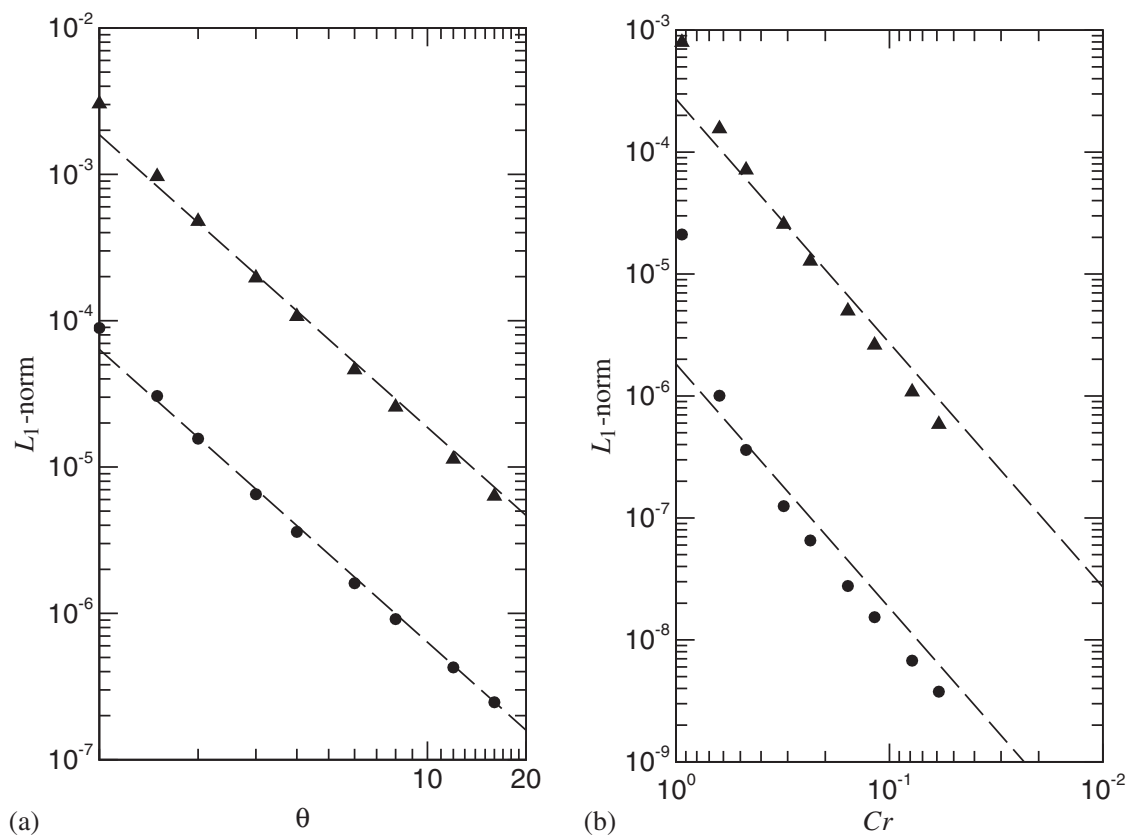

Figure 14. Discretization errors for the propagation of a solitary wave. Ideal second-order slope (- -); numerical errors for $\varepsilon=0.1(\bullet)$; and numerical errors for $\varepsilon=0.6(\boldsymbol{\Lambda})$. Convergence for (a) mesh refinement and (b) time-step refinement.

\subsection{Closed basin oscillation}

We analyse the convergence of the scheme and its conservation properties for the oscillation of a closed flat basin.

For the convergence test, the basin is $9 \mathrm{~m}$ long and $0.45 \mathrm{~m}$ deep. The boundaries are closed, hence the boundary conditions are $\bar{u}(-4.5, t)=\bar{u}(4.5, t)=0$. The initial condition is a free-surface Gaussian-shaped hump, with null velocity in the whole domain. The hump is centred at $x_{0}$

$$
\zeta(x)=a \exp \left[-\frac{1}{2 \mu^{2}}\left(x-x_{0}\right)^{2}\right]
$$

where $a$ is the hump amplitude, $\mu=0.5 \mathrm{~m}$ is the shape parameter, and $x_{0}=0$. The behaviour of the solution is analysed for two waves: a weakly non-linear wave with $a=0.045 \mathrm{~m}$, hence $\varepsilon=0.1$; a strongly non-linear wave with $a=0.225 \mathrm{~m}$, hence $\varepsilon=0.5$. The linear oscillation modes of the basin have wave numbers

$$
k_{n}=\frac{n \pi}{L} \quad \text { for } n=1 \ldots \infty
$$

where $L$ is the basin length. The natural frequencies, $\omega_{n}$, are given by the Boussinesq dispersion relation:

$$
\omega_{n}^{2}=g k_{n} \frac{k_{n} h_{0}}{1+\left(k_{n} h_{0}\right)^{2} / 3}
$$



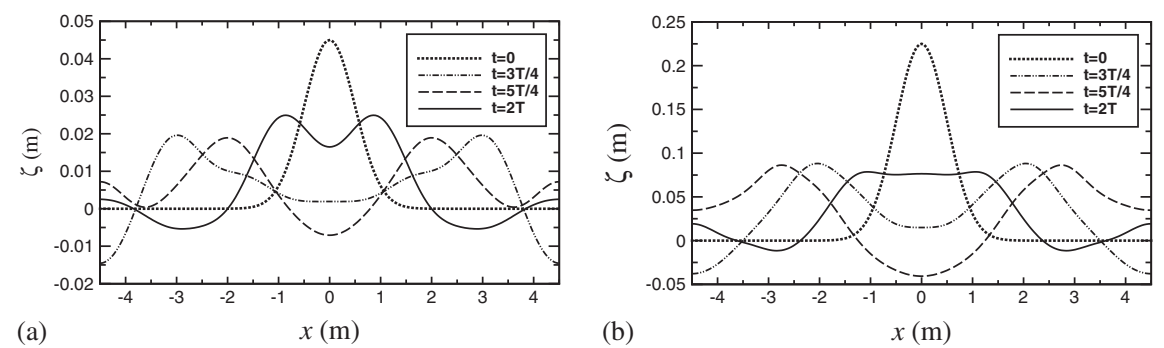

Figure 15. Closed basin free-surface profiles: (a) $\varepsilon=0.1$ and (b) $\varepsilon=0.5$.

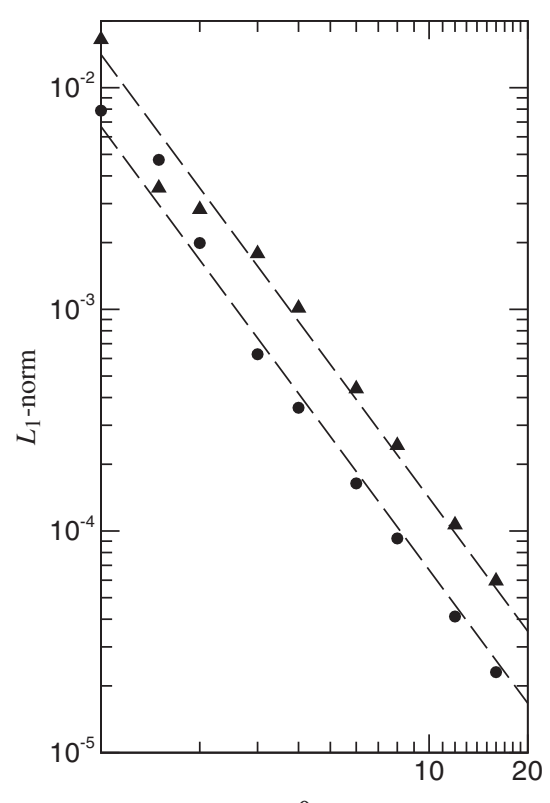

(a)

$\theta$

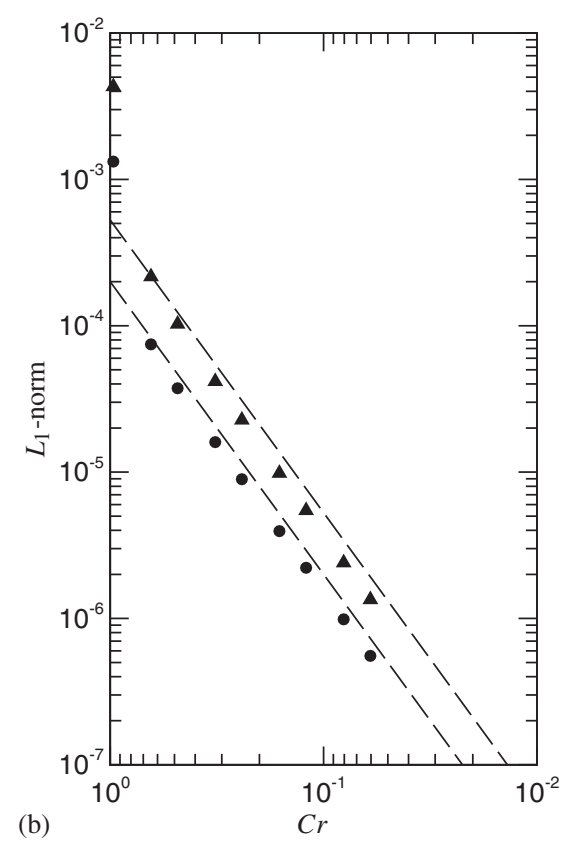

Figure 16. Discretization errors for the oscillation of a closed basin. Ideal second-order slope $(--)$; numerical errors for $\varepsilon=0.1(\bullet)$; and numerical errors for $\varepsilon=0.5(\boldsymbol{\Lambda})$. Convergence for (a) mesh refinement and (b) time-step refinement.

where $h_{0}$ is the basin depth. The lowest resonance period, corresponding to the first symmetric mode, $n=2$, is $T_{2}=4.3534 \mathrm{~s}$. The simulations were run up to $t=9 \mathrm{~s} \approx 2 T_{2}$. In Figure 15 the freesurface profiles are shown when a regular finite element mesh with $\Delta x=0.045 \mathrm{~m}$ and a time step $\Delta t=0.017 \mathrm{~s}(C r=0.79$ and $\theta=10)$ is used.

The spatial discretization convergence was analysed using a fixed time step $\Delta t=0.005 \mathrm{~s}$ and two geometric sequences for $\Delta x: \Delta x=0.45 / 2^{n-1} \mathrm{~m}, n=1 \ldots 6$, and $\Delta x=0.3 / 2^{n-1} \mathrm{~m}, n=1 \ldots 5$. Results are presented in Figure 16(a). For the weakly non-linear wave, the mean convergence rate for spatial discretization is $\bar{r}=2.18$, while for the strongly non-linear wave it is $\bar{r}=1.88$. The ideal slope for a second-order spatial discretization convergence is well recovered, except for the 
A PETROV-GALERKIN FEM FOR THE BOUSSINESQ WAVE MODEL
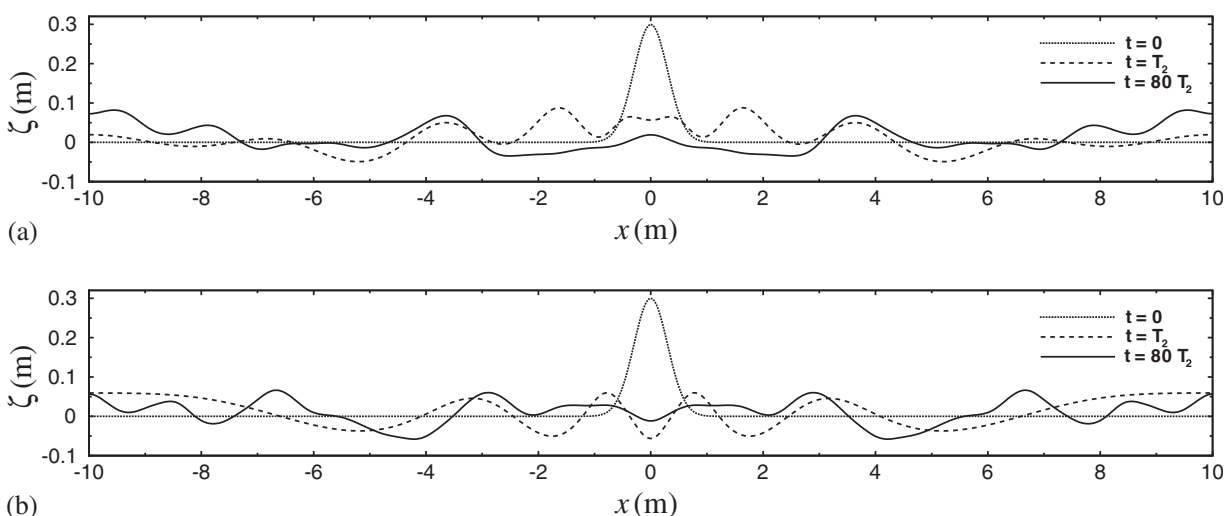

Figure 17. Closed basin free-surface profiles: (a) coarse mesh and (b) refined mesh.

strongly non-linear wave in a very coarse mesh with $\theta=1$. Removal of this mesh from the analysis improves the Cauchy convergence rate to $\bar{r}^{*}=2.03$. The spatial discretization is clearly $\mathcal{O}\left(\Delta x^{2}\right)$ accurate.

The convergence test was also performed for time discretization. A constant mesh size, $\Delta x=$ $0.075 \mathrm{~m}$, hence $\theta=6$, was used, along with two geometric sequences for $\Delta t: \Delta t=0.0345 / 2^{n-1} \mathrm{~s}$, $n=1 \ldots 6$, and $\Delta t=0.023 / 2^{n-1} \mathrm{~s}, n=1 \ldots 5$. Results are presented in Figure $16(\mathrm{~b})$. The secondorder ideal slope is well recovered except for the first time step size, for which $\mathrm{Cr}=0.97$, which might be too close to the scheme stability limit. The computed mean convergence rates were $\bar{r}=2.49$ and 2.59 for $\varepsilon=0.1$ and 0.6 , respectively. These values were corrected to $\bar{r}^{*}=2.05$ and 2.12 after removal of the results for the coarser time step size, confirming that the time discretization error is $\mathcal{O}\left(\Delta t^{2}\right)$.

As previously shown in Section 2.2, the present Boussinesq model is not energy conservative. Nevertheless, in the closed basin problem the extra terms in (12a) have an oscillating behaviour and some averaged energy conservation behaviour might be displayed. Let us consider again the basin oscillation case for an initial Gaussian hump located at its centre. Following Reference [27] the basin length was set to $L=20 \mathrm{~m}$ with depth $h_{0}=1 \mathrm{~m}$. The shape parameter was set to $\mu=0.3 \mathrm{~m}$ and the amplitude was $a=0.3 \mathrm{~m}$, thus ensuring some non-linearity, $\varepsilon=0.3$, while dispersion will also have an influence. Two numerical discretizations were used: $\Delta x_{1}=0.05 \mathrm{~m}$ and $\Delta t_{1}=0.01268 \mathrm{~s}$; and $\Delta x_{2}=0.025 \mathrm{~m}$ and $\Delta t_{2}=0.00634 \mathrm{~s}$. Thus, $C r_{1}=C r_{2}=0.794$, and $\theta_{1}=20$ and $\theta_{2}=40$. The basin's first symmetric resonance mode is $T_{2}=6.490 \mathrm{~s}$, and the simulation was run up to $t=40960 \Delta t \approx 80 T_{2}$. During the simulations, non-linearity and dispersion will favour the wave-wave interaction and the redistribution of energy at several wave numbers, thus allowing the observation of the effect of the proposed dissipation mechanism.

Again the scheme showed very good mass conservation properties. In both runs wave mass was preserved: a constant figure (up to nine significant digits) was registered.

A fine discretization is necessary for good wave profile description. Comparison of the freesurface profiles for both meshes shows that already at $t \approx T_{2}$ (see Figure 17) the short length features of the wave are not well reproduced by the coarser mesh. This might be due to a stronger energy dissipation on this mesh.

Time histories of total energy are depicted in Figure 18. The registered high-frequency oscillations in total energy are an intrinsic property of Boussinesq model and do not result from any 


\section{P. AVILEZ-VALENTE AND F. J. SEABRA-SANTOS}

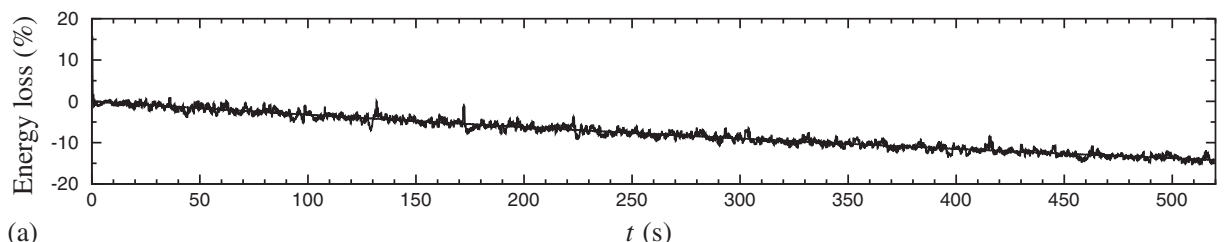

(a)

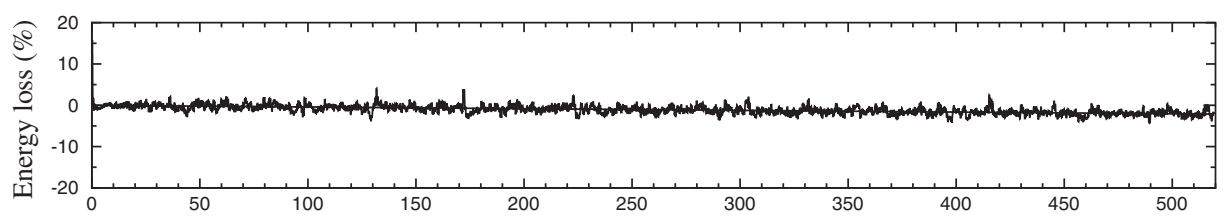

(b)

$t(\mathrm{~s})$

Figure 18. Time history of total energy: (a) coarse mesh and (b) refined mesh.

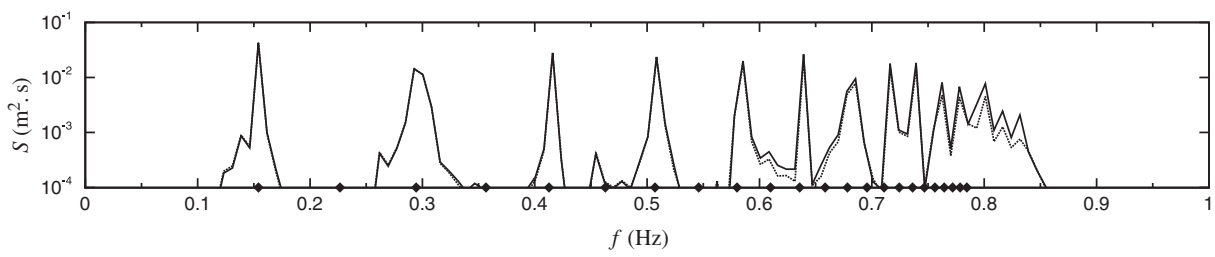

Figure 19. Power density spectra of free surface time series at $x=0$. Coarse mesh $(--)$; refined mesh $(-)$; and linear resonance modes $(\diamond)$.

feature of the numerical scheme. The least-squares method was used to find a best fit for a linear mean energy dissipation rate. For the coarse mesh, mean energy dissipation stands about $14 \%$ after $519 \mathrm{~s}$, while for the refined mesh dissipation is reduced to just $2.1 \%$.

In Figure 19 the power density spectra obtained at $x=0$ are shown (averaged for four timeseries of length $\approx 20 T_{2}$ ). It is clear that the basin oscillates with its natural frequencies (symmetric modes only). The first 24 modes are identified in the figure. The modes higher than the 24th lay in the region $[0.79,0.86] \mathrm{Hz}$ and are not identified in the figure. The dissipation mechanism prevents energy from concentrating on the shortest wavelengths, thus inhibiting the blow-up of the simulations. Comparison of the results for the coarse and the refined meshes clearly shows that the third-order dissipation mechanism only affects the highest wavelengths: those with frequencies above $0.5 \mathrm{~Hz}$ on this case, and that only the frequencies above $0.7 \mathrm{~Hz}$ show a significant decrease in energy density.

\subsection{Closed non-flat basin oscillation}

Let us consider again a $9 \mathrm{~m}$ long basin. The combined effect of a sinusoidal-like bottom shape and of a non-uniform finite element mesh upon convergence will be considered. The basin depth is $0.45 \mathrm{~m}$ at the deepest points and $0.225 \mathrm{~m}$ at the shallowest one (see Figure 20).

The initial condition is the free-surface Gaussian-shaped hump described by Equation (70) and centred at $x_{0}=0$, with amplitude $a=0.09 \mathrm{~m}$, and shape parameter $\mu=0.5 \mathrm{~m}$. Initial velocity is null 
A PETROV-GALERKIN FEM FOR THE BOUSSINESQ WAVE MODEL

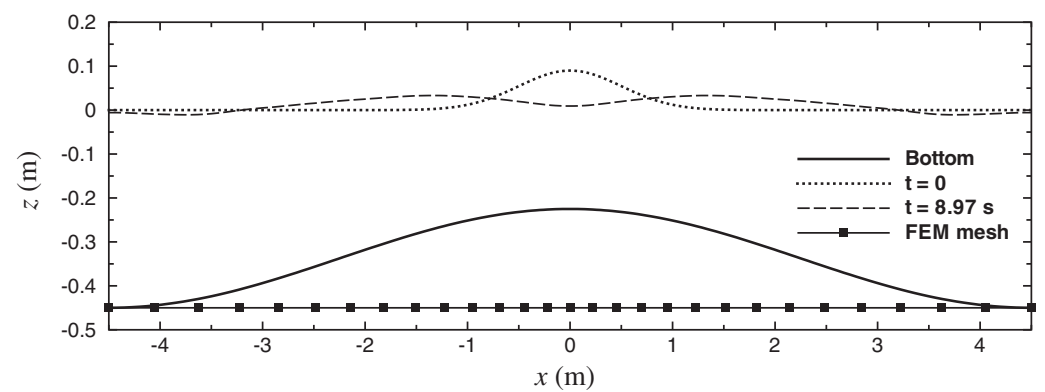

Figure 20. Bottom, FEM mesh, and free-surface profiles for sinus like bottom shape basin.

all over the domain. The non-linearity parameter varies from $\varepsilon=0.2$ at $x= \pm 4.5 \mathrm{~m}$ to $\varepsilon=0.4$ at $x=0$. Within each time step, one predictor + two corrector steps were used.

The spatial discretization convergence was analysed using two sequences of non-uniform meshes. For the first sequence, the initial mesh was such that $0.218 \mathrm{~m} \leqslant \Delta x_{1} \leqslant 0.45 \mathrm{~m}$ (cf. Figure 20), and the geometric sequence was $\Delta x=\Delta x_{1} / 2^{n-1} \mathrm{~m}, n=1 \ldots 6$. For the second sequence, the initial mesh was such that $0.145 \mathrm{~m} \leqslant \Delta x_{1} \leqslant 0.3 \mathrm{~m}$, and the geometric sequence was $\Delta x=\Delta x_{1} / 2^{n-1} \mathrm{~m}, n=1 \ldots .5$. The results are represented in Figure 21(a). The mean convergence rate for spatial discretization is $\bar{r}=1.84$. The ideal slope for second-order convergence is well recovered, but for the very coarse mesh $(\theta=1)$. Removal of this mesh from the analysis improves the Cauchy convergence rate to $\bar{r}^{*}=1.97$, showing that a $\mathcal{O}\left(\Delta x^{2}\right)$ accuracy is achieved.

The convergence rate was also analysed for the time discretization. A non-uniform mesh such that $0.03625 \mathrm{~m} \leqslant \Delta x \leqslant 0.075 \mathrm{~m}$ was used, along with two geometric sequences for $\Delta t: \Delta t=$ $0.01725 / 2^{n-1} \mathrm{~s}, n=1 \ldots 6$, and $\Delta t=0.0115 / 2^{n-1} \mathrm{~s}, n=1 \ldots 5$. The ideal slope for second-order convergence is well recovered. The results are represented in Figure 21(b). The mean convergence rate for time discretization is $\bar{r}=1.99$, confirming the $\mathcal{O}\left(\Delta t^{2}\right)$ accuracy. These results allow us to conclude that a non-flat bottom or a non-uniform mesh does not affect the convergence rate of the proposed finite element scheme.

\subsection{Wave propagation over a submerged bar}

We simulate the propagation of a periodic sinusoidal wave over a submerged bar. The numerical results for both the proposed PG scheme and a BG scheme for which the weighting function coefficients, $\alpha$ and $\beta$ are identically null are compared with experimental data [46].

Water depth is $0.86 \mathrm{~m}$ away from the bar and $0.20 \mathrm{~m}$ over the bar crest. The numerical channel is $43 \mathrm{~m}$ long. The wave amplitude is $a=0.02 \mathrm{~m}$, the wave period is $T=2.857 \mathrm{~s}$, and the wavelength is $\lambda=7.709 \mathrm{~m}$ according to Airy's linear theory (or $\lambda=7.689 \mathrm{~m}$, according to the Boussinesq model). $\Delta x=0.1 \mathrm{~m}$ and $\Delta t=1 / 30 \mathrm{~s}$, so that $\theta=8.6, C r=0.97$ and $k h=0.70 \approx \pi / 5$ at the wave inlet, thus in the Boussinesq validity range, and $\theta=2$ and $C r=0.47$ over the bar crest.

Eleven wave gauges were used (cf. Table II and Figure 22). The boundary conditions were: on the seaward side

$$
\hat{\zeta}(0, t)=\zeta_{1}^{*}(0, t) \quad \text { and } \quad \hat{u}(0, t)=c \frac{\zeta_{1}^{*}(0, t)}{h+\zeta_{1}^{*}(0, t)}
$$



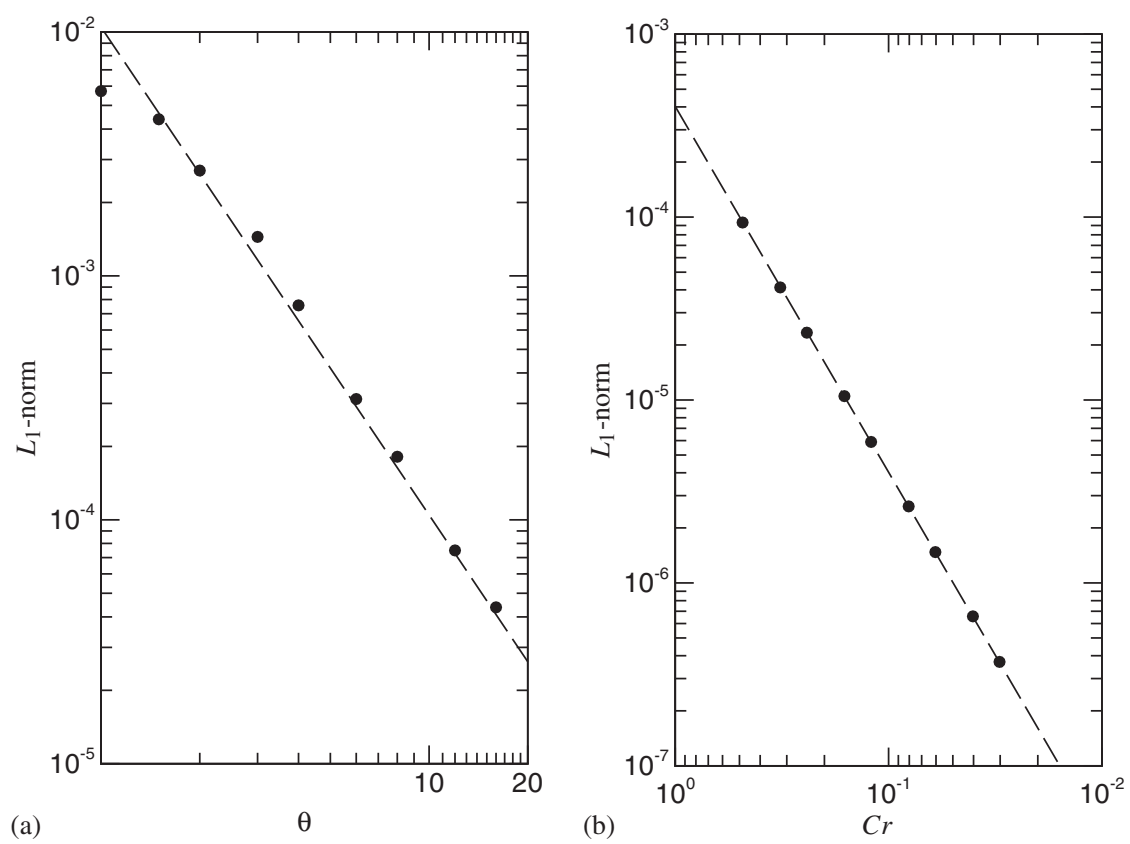

Figure 21. Discretization errors for the oscillation of a closed basin with a sinusoidal like bottom shape. Ideal second-order slope $(--)$ and numerical errors $(\bullet)$. Convergence for (a) mesh refinement and (b) time-step refinement.

Table II. Position of the wave gauges.

\begin{tabular}{|c|c|c|c|c|c|c|c|c|c|c|c|}
\hline $\begin{array}{l}\text { Gauge } \\
x(\mathrm{~m})\end{array}$ & $\begin{array}{l}1 \\
0.0\end{array}$ & $\begin{array}{l}2 \\
4.0\end{array}$ & $\begin{array}{l}3 \\
6.4\end{array}$ & $\begin{array}{c}4 \\
17.0\end{array}$ & $\begin{array}{c}5 \\
21.0\end{array}$ & $\begin{array}{c}6 \\
23.0\end{array}$ & $\begin{array}{c}7 \\
25.0\end{array}$ & $\begin{array}{c}8 \\
27.4\end{array}$ & $\begin{array}{c}9 \\
30.6\end{array}$ & $\begin{array}{l}10 \\
34.0\end{array}$ & $\begin{array}{l}11 \\
38.0\end{array}$ \\
\hline
\end{tabular}

where $\zeta_{1}^{*}$ stands for the experimental signal at Gauge 1; on the leeward side, a radiation condition was imposed

$$
\hat{u}(43, t)=c \frac{\hat{\zeta}(43, t)}{h+\hat{\zeta}(43, t)}
$$

At both boundaries, $c$ is the wave velocity for $T=2.857 \mathrm{~s}$ according to the Boussinesq dispersion relation (6).

During propagation on the seaward side of the bar, the wave is essentially made up of only one harmonic. At the upward slope, higher harmonics are generated due to the increasing non-linear interaction. Over the bar crest dispersion and non-linearity effects are in balance, and the different harmonics become locked and propagate in phase in a solitary wave fashion. These harmonics are quickly set free on the downward slope, where dispersion dominates.

In Figure 23, the non-dissipative and oscillation prone character of the BG scheme is clearly shown: a very shortlength non-propagating disturbance is polluting the numerical solution. On the 
A PETROV-GALERKIN FEM FOR THE BOUSSINESQ WAVE MODEL

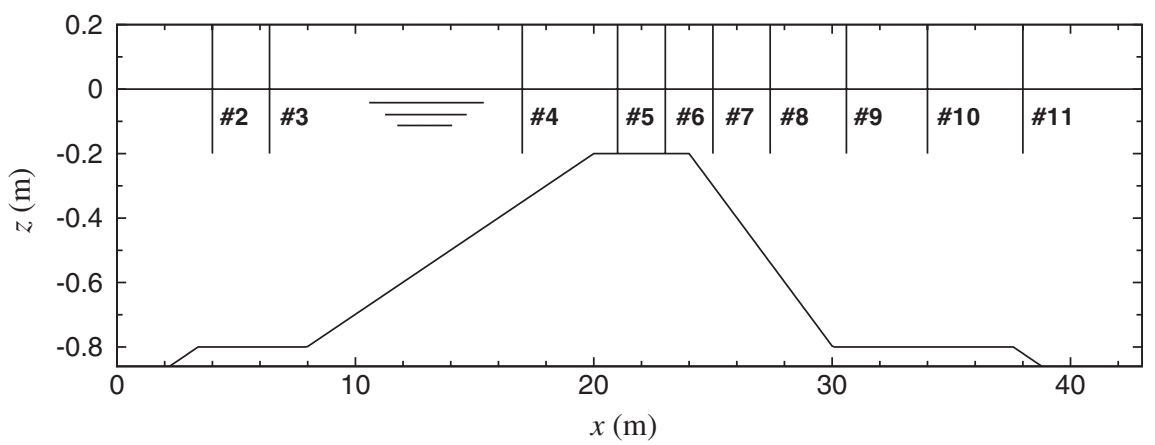

Figure 22. Layout of the submerged bar experiment.

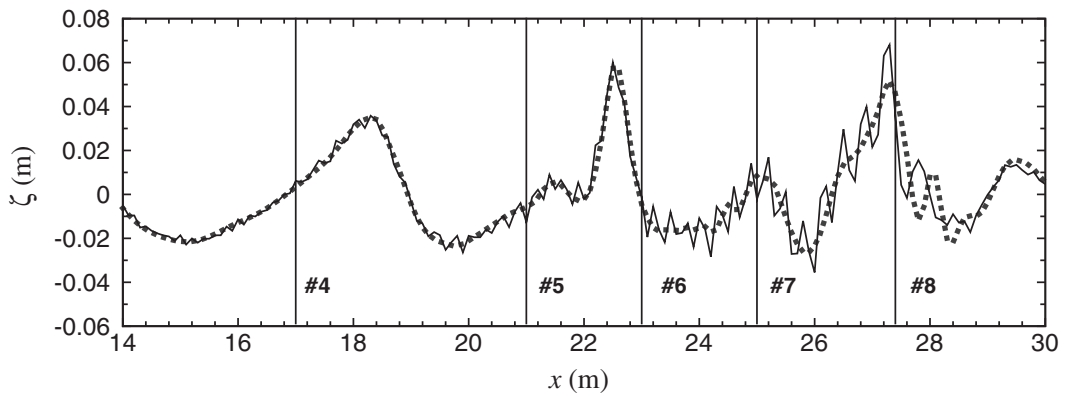

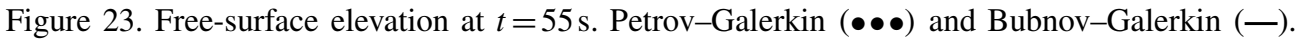

other hand, the PG solution does not display any such numerical noise, while still capturing the main wave features.

Figure 24 displays the comparison of time histories of the free-surface elevation between numerical results and experimental data at Gauges 4-6 and 9-11. A small phase correction due to the $\beta$-coefficient is apparent in the PG solution from Gauge 5 downwards. Wave phase and amplitude are well captured, except for the last three gauges. Spectral analysis (see Figure 25) shows us that for Gauges 9-11 the third and higher harmonics are not reproduced. This is due to the fact that these harmonics have frequencies higher than $0.93 \mathrm{~Hz}$ which, for $h=0.86 \mathrm{~m}$, is the asymptotic limit of the Boussinesq equations (the limit for the discrete equations is even lower). On the other hand, the second component, for which $T=1.428 \mathrm{~s}$, is actually larger than the first component at the outward boundary. This might lead to an error of at most $3.6 \%$ in the wave velocity in boundary condition (73b). However, tests performed using a domain long enough for boundary reflection not to occur showed this error to be negligible.

\section{CONCLUSIONS}

We presented a PG method for the one-dimensional depth-integrated weakly non-linear and dispersive Boussinesq wave equations. Space-time finite elements are used for a full discretization. The 

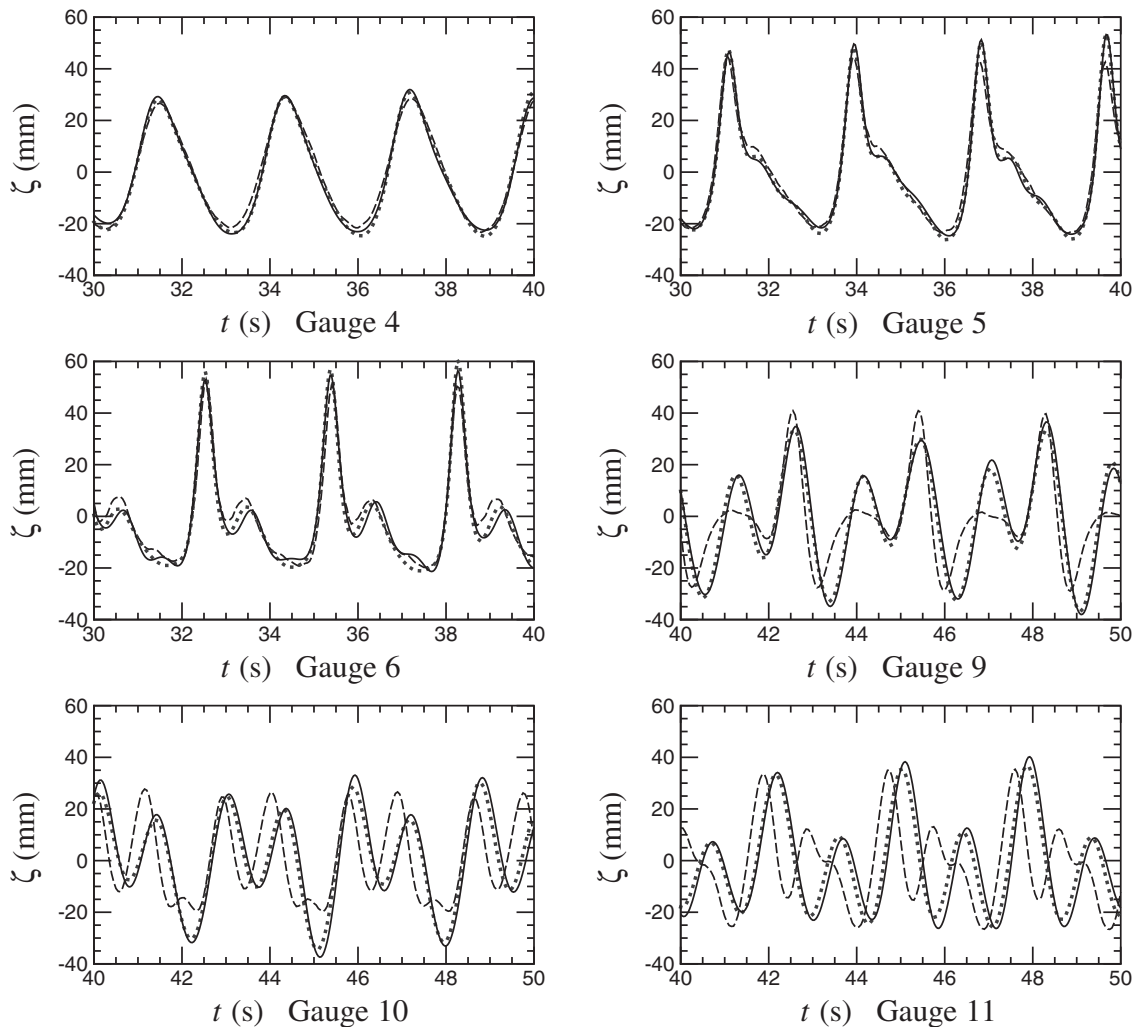

Figure 24. Wave gauge time histories. Experimental data (- $)$ and numerical results: PG $(\bullet \bullet \bullet)$ and BG (一).

shape functions are bilinear in space and time, and the weighting functions are linear in space and quadratic in time. Both the shape and the weighting functions have $C^{0}$-continuity. Inclusion of extra upwind terms in the weighting functions allowed for dispersion correction and for the introduction of a high-order dissipation mechanism. Accuracy and stability were deeply investigated using numerical analysis: truncation analysis and von Neumann's spectral analysis showed the scheme to be fourth-order phase accurate with a third-order highly selective dissipation mechanism. Four applications were addressed that showed the numerical scheme to be able to accurately reproduce the expected features of the Boussinesq model for solitary wave propagation, closed basin oscillation for both a flat bottom and a non-flat bottom basin, and propagation of a periodic wave over a submerged bar. In the latter case study, laboratory experiments were used for comparison and the Boussinesq model shortcomings became immediately evident. A convergence analysis showed the scheme to possess a full second-order convergence rate for both element size and time-step discretization. The system matrix is symmetric and constant throughout the simulations. This is an important feature of the scheme because extensions in two horizontal dimensions with relatively large-scale domains to be discretized will be affordable in terms of computational cost. An extension of the scheme to two horizontal dimensions, using bilinear quadrilateral unstructured meshes in space, can be obtained, based on the weighting function suggested in Reference [47]. 
A PETROV-GALERKIN FEM FOR THE BOUSSINESQ WAVE MODEL
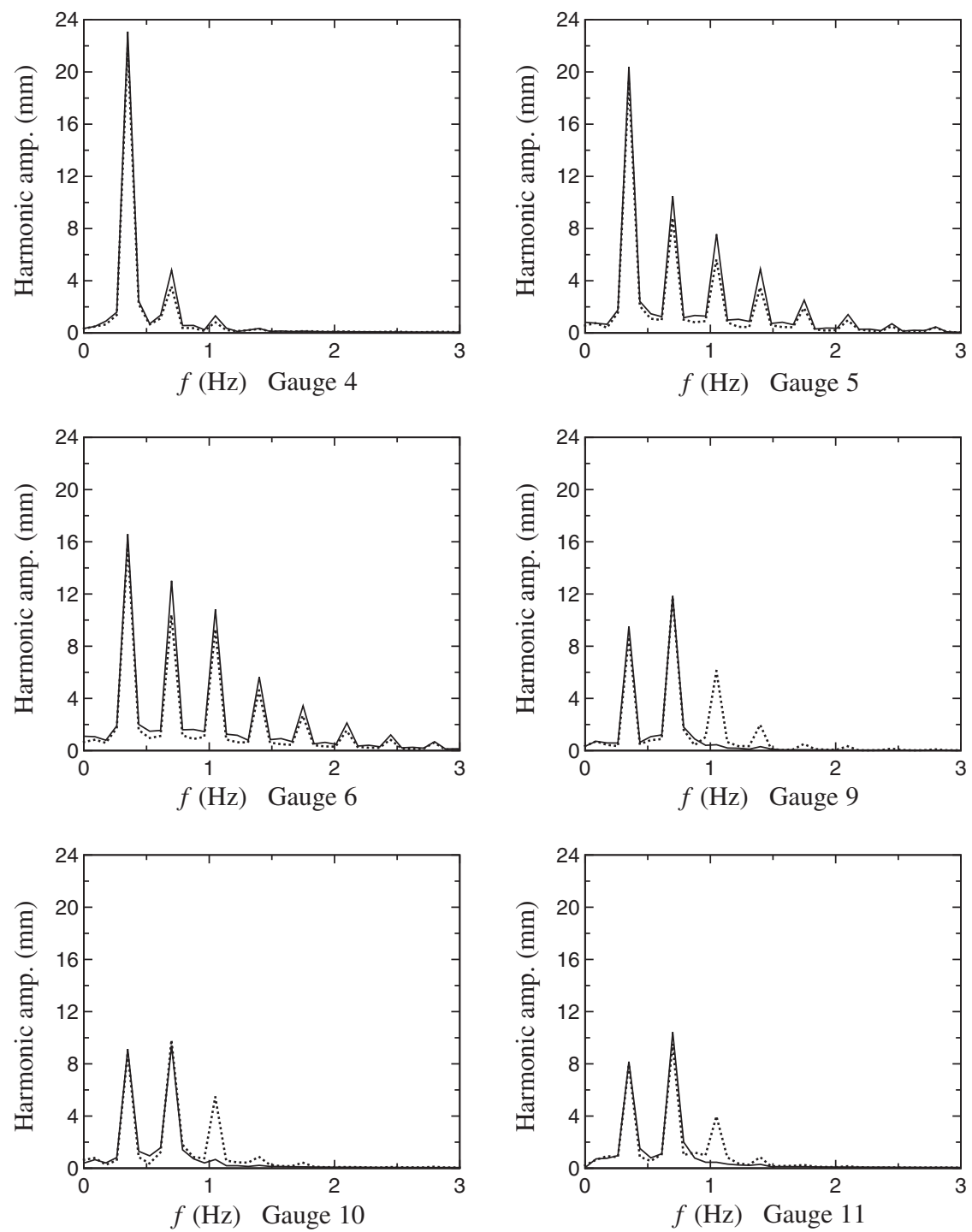

Figure 25. Amplitude spectra of experimental data $(\cdots)$ and numerical PG results (-).

Nevertheless, special care must be taken due to the presence of cross derivatives in the stabilizing term. An application of a similar scheme to the enhanced Boussinesq models is being investigated, although accuracy might still be restricted to the third order.

\section{APPENDIX A}

For a non-flat bottom, the derivation of the equivalent differential equations is slightly more complex, but a system of equations with a third-order truncation error can also obtained. 


\section{P. AVILEZ-VALENTE AND F. J. SEABRA-SANTOS}

Let us write the non-dimensional counterpart of Equations (23):

$$
\begin{gathered}
\mathbf{M} \Delta \zeta=-\frac{\Delta t}{2} \mathbf{C}\left(\mathbf{p}^{(n+1)}+\mathbf{p}^{(n)}\right)+\beta \frac{\Delta x \Delta t}{4 \sqrt{h}} \mathbf{K}_{h} \Delta \mathbf{q} \\
\left(\mathbf{M}+\sigma^{2} \mathbf{K}_{\sigma}\right) \Delta \mathbf{u}=- \\
-\frac{\Delta t}{2}\left[\mathbf{C}\left(\mathbf{q}^{(n+1)}+\mathbf{q}^{(n)}\right)+\alpha \frac{\Delta x}{\sqrt{h}} \mathbf{K}\left(\mathbf{p}^{(n+1)}+\mathbf{p}^{(n)}\right)\right] \\
+\beta_{\ell} \mathbf{C}_{h}^{*} \Delta \mathbf{u}-\alpha \frac{\Delta x}{\sqrt{h}} \mathbf{C}^{\mathrm{T}} \Delta \zeta+\beta \frac{\Delta x \Delta t}{4 \sqrt{h}} \mathbf{K} \Delta \mathbf{p}
\end{gathered}
$$

In Equations (A1), $\bar{h}$ is the element dimensionless mean depth, while the dimensionless momentum and wave energy densities are

$$
\hat{p}=(h+\varepsilon \hat{\zeta}) \hat{u} \quad \text { and } \quad \hat{q}=\varepsilon \frac{\hat{u}^{2}}{2}+\hat{\zeta}
$$

and matrix $\mathbf{C}_{h}^{*}$ has entries

$$
\left(C_{h}^{*}\right)_{i j}=\frac{1}{3} \sum_{k=1}^{2} \sum_{\ell=1}^{2}\left\langle N_{i} N_{k} \frac{\mathrm{d} N_{\ell}}{\mathrm{d} x^{*}} \frac{\mathrm{d} N_{j}}{\mathrm{~d} x}\right\rangle h_{k} h_{\ell}
$$

Assuming a regular finite element mesh and assembling Equations (A1) over the entire computational domain lead us to the following discrete equations for the $i$ th node away from the boundaries:

$$
\begin{aligned}
\mathscr{L}_{\zeta} \equiv & \frac{\Delta x}{6}\left[\left(\hat{\zeta}_{i+1}^{(n+1)}+4 \hat{\zeta}_{i}^{(n+1)}+\hat{\zeta}_{i-1}^{(n+1)}\right)-\left(\hat{\zeta}_{i+1}^{(n)}+4 \hat{\zeta}_{i}^{(n)}+\hat{\zeta}_{i-1}^{(n)}\right)\right] \\
& +\frac{\Delta t}{4}\left\{\left[\left(h_{i+1}+\varepsilon \hat{\zeta}_{i+1}^{(n+1)}\right) \hat{u}_{i+1}^{(n+1)}-\left(h_{i-1}+\varepsilon \hat{\zeta}_{i-1}^{(n+1)}\right) \hat{u}_{i-1}^{(n+1)}\right]\right. \\
& \left.+\left[\left(h_{i+1}+\varepsilon \hat{\zeta}_{i+1}^{(n)}\right) \hat{u}_{i+1}^{(n)}-\left(h_{i-1}+\varepsilon \hat{\zeta}_{i-1}^{(n)}\right) \hat{u}_{i-1}^{(n)}\right]\right\} \\
& -\frac{\Delta t^{2}}{24 \Delta x}\left[\left(h_{i-1}+h_{i}\right)\left(\hat{\zeta}_{i-1}^{(n+1)}-\hat{\zeta}_{i-1}^{(n)}\right)-\left(h_{i-1}+2 h_{i}+h_{i+1}\right)\left(\hat{\zeta}_{i}^{(n+1)}-\hat{\zeta}_{i}^{(n)}\right)\right. \\
& \left.+\left(h_{i}+h_{i+1}\right)\left(\hat{\zeta}_{i+1}^{(n+1)}-\hat{\zeta}_{i+1}^{(n)}\right)\right] \\
& -\frac{\varepsilon \Delta t^{2}}{48 \Delta x}\left\{\left(h_{i-1}+h_{i}\right)\left[\left(\hat{u}_{i-1}^{(n+1)}\right)^{2}-\left(\hat{u}_{i-1}^{(n)}\right)^{2}\right]-\left(h_{i-1}+2 h_{i}+h_{i+1}\right)\left[\left(\hat{u}_{i}^{(n+1)}\right)^{2}-\left(\hat{u}_{i}^{(n)}\right)^{2}\right]\right. \\
& \left.+\left(h_{i}+h_{i+1}\right)\left[\left(\hat{u}_{i+1}^{(n+1)}\right)^{2}-\left(\hat{u}_{i+1}^{(n)}\right)^{2}\right]\right\}=0
\end{aligned}
$$

and

$$
\begin{aligned}
\mathscr{L}_{\bar{u}} \equiv & \frac{\Delta x}{6}\left[\left(\hat{u}_{i+1}^{(n+1)}+4 \hat{u}_{i}^{(n+1)}+\hat{u}_{i-1}^{(n+1)}\right)-\left(\hat{u}_{i+1}^{(n)}+4 \hat{u}_{i}^{(n)}+\hat{u}_{i-1}^{(n)}\right)\right] \\
& +\varepsilon \frac{\Delta t}{4}\left\{\left[\left(\hat{u}_{i+1}^{(n+1)}\right)^{2}-\left(\hat{u}_{i-1}^{(n+1)}\right)^{2}\right]+\left[\left(\hat{u}_{i+1}^{(n)}\right)^{2}-\left(\hat{u}_{i-1}^{(n)}\right)^{2}\right]\right\}
\end{aligned}
$$


A PETROV-GALERKIN FEM FOR THE BOUSSINESQ WAVE MODEL

$$
\begin{aligned}
& +\frac{\Delta t}{4}\left[\left(\hat{\zeta}_{i+1}^{(n+1)}-\hat{\zeta}_{i-1}^{(n+1)}\right)+\left(\hat{\zeta}_{i+1}^{(n)}-\hat{\zeta}_{i-1}^{(n)}\right)\right] \\
& -\frac{\sigma^{2}}{9 \Delta x}\left[\left(h_{i-1}^{2}+h_{i-1} h_{i}+h_{i}^{2}\right)\left(\hat{u}_{i-1}^{(n+1)}-\hat{u}_{i-1}^{(n)}\right)\right. \\
& -\left(h_{i-1}^{2}+h_{i-1} h_{i}+2 h_{i}^{2}+h_{i} h_{i+1}+h_{i+1}^{2}\right)\left(\hat{u}_{i}^{(n+1)}-\hat{u}_{i}^{(n)}\right) \\
& \left.+\left(h_{i}^{2}+h_{i} h_{i+1}+h_{i+1}^{2}\right)\left(\hat{u}_{i+1}^{(n+1)}-\hat{u}_{i+1}^{(n)}\right)\right] \\
& -\alpha_{(1)}^{\prime} \frac{\Delta t^{2}}{2 \Delta x}\left[2 h_{i-1}+\varepsilon\left(\hat{\zeta}_{i-1}^{(n+1)} \hat{u}_{i-1}^{(n+1)}+\hat{\zeta}_{i-1}^{(n)} \hat{u}_{i-1}^{(n)}\right)\right] \\
& +\left(\alpha_{(1)}^{\prime}+\alpha_{(2)}^{\prime}\right) \frac{\Delta t^{2}}{2 \Delta x}\left[2 h_{i}+\varepsilon\left(\hat{\zeta}_{i}^{(n+1)} \hat{u}_{i}^{(n+1)}+\hat{\zeta}_{i}^{(n)} \hat{u}_{i}^{(n)}\right)\right] \\
& -\alpha_{(2)}^{\prime} \frac{\Delta t^{2}}{2 \Delta x}\left[2 h_{i+1}+\varepsilon\left(\hat{\zeta}_{i+1}^{(n+1)} \hat{u}_{i+1}^{(n+1)}+\hat{\zeta}_{i+1}^{(n)} \hat{u}_{i+1}^{(n)}\right)\right] \\
& +\frac{\Delta t}{2}\left[\alpha_{(1)}^{\prime}\left(\hat{\zeta}_{i+1}^{(n+1)}-\hat{\zeta}_{i+1}^{(n)}\right)+\left(\alpha_{(1)}^{\prime}-\alpha_{(2)}^{\prime}\right)\left(\hat{\zeta}_{i}^{(n+1)}-\hat{\zeta}_{i}^{(n)}\right)-\alpha_{(2)}^{\prime}\left(\hat{\zeta}_{i-1}^{(n+1)}-\hat{\zeta}_{i-1}^{(n)}\right)\right] \\
& -\frac{\sigma^{2}}{18 \Delta x}\left[\left(h_{i-1}^{2}+h_{i-1} h_{i}-h_{i}^{2}\right)\left(u_{i-1}^{(n+1)}-u_{i-1}^{(n)}\right)\right. \\
& -\left(h_{i-1}^{2}+h_{i-1} h_{i}-2 h_{i}^{2}+h_{i} h_{i+1}+h_{i+1}^{2}\right)\left(u_{i}^{(n+1)}-u_{i}^{(n)}\right) \\
& \left.+\left(-h_{i}^{2}+h_{i} h_{i+1}+h_{i+1}^{2}\right)\left(u_{i+1}^{(n+1)}-u_{i+1}^{(n)}\right)\right] \\
& +\frac{\Delta t^{2}}{12 \Delta x}\left\{\left[\left(h_{i+1}+\varepsilon \hat{\zeta}_{i+1}^{(n+1)}\right) \hat{u}_{i+1}^{(n+1)}-2\left(h_{i}+\varepsilon \hat{\zeta}_{i}^{(n+1)}\right) \hat{u}_{i}^{(n+1)}+\left(h_{i-1}+\varepsilon \hat{\zeta}_{i-1}^{(n+1)}\right) \hat{u}_{i-1}^{(n+1)}\right]\right. \\
& \left.-\left[\left(h_{i+1}+\varepsilon \hat{\zeta}_{i+1}^{(n)}\right) \hat{u}_{i+1}^{(n)}-2\left(h_{i}+\varepsilon \hat{\zeta}_{i}^{(n)}\right) \hat{u}_{i}^{(n)}+\left(h_{i-1}+\varepsilon \hat{\zeta}_{i-1}^{(n)}\right) \hat{u}_{i-1}^{(n)}\right]\right\}=0
\end{aligned}
$$

where $\hat{\zeta}_{i}^{(n)}=\hat{\zeta}\left(x_{i}, t_{n}\right), \hat{u}_{i}^{(n)}=\hat{u}\left(x_{i}, t_{n}\right),\left(\hat{u}_{i}^{(n)}\right)^{2}=\left(\hat{u}\left(x_{i}, t_{n}\right)\right)^{2}$, and $h_{i}=h\left(x_{i}\right)$. The coefficients $\alpha_{(1)}^{\prime}$ and $\alpha_{(2)}^{\prime}$ are defined as $\alpha / C r$ at the neighbouring elements, i.e.

$$
\alpha_{(1)}^{\prime}=\frac{\left(h_{i-1}+h_{i}\right) \Delta t^{2}}{2 \Delta x^{2}-\left(h_{i-1}+h_{i}\right) \Delta t^{2}} \quad \text { and } \quad \alpha_{(2)}^{\prime}=\frac{\left(h_{i}+h_{i+1}\right) \Delta t^{2}}{2 \Delta x^{2}-\left(h_{i}+h_{i+1}\right) \Delta t^{2}}
$$

The equivalent differential equations for these algebraic equations are a third-order approximation of the Boussinesq equations for mild bottom curvature (8a) and (10a), provided that $\varepsilon \sim \sigma^{2} \sim \beta_{\ell}^{2} \sim \Delta x^{2} \sim \Delta t^{2}$ :

$$
\frac{\partial \hat{\zeta}}{\partial t}+\frac{\partial}{\partial x}[(h+\varepsilon \hat{\zeta}) \hat{u}]=\mathcal{O}\left(\Delta x^{4}, \varepsilon \Delta x^{2}, \sigma^{2} \Delta x^{2}, \beta_{\ell} \Delta x^{4}, \beta_{\ell} \varepsilon \Delta x^{2}, \beta_{\ell} \sigma^{2} \Delta x^{2}, \beta_{\ell}^{3} \Delta x^{2}\right)
$$




\section{P. AVILEZ-VALENTE AND F. J. SEABRA-SANTOS}

and

$$
\begin{gathered}
\frac{\partial \hat{u}}{\partial t}+\varepsilon \hat{u} \frac{\partial \hat{u}}{\partial x}+\frac{\partial \hat{\zeta}}{\partial x}-\sigma^{2}\left[\frac{\partial}{\partial x}\left(\frac{h^{2}}{3} \frac{\partial^{2} \hat{u}}{\partial x \partial t}\right)+\beta_{\ell} \frac{h}{3} \frac{\mathrm{d} h}{\mathrm{~d} x^{*}} \frac{\partial^{2} \hat{u}}{\partial x \partial t}\right] \\
=-\frac{\Delta x^{3}}{12} \operatorname{Cr}^{3} \sqrt{h} \frac{\partial^{4} \hat{u}}{\partial x^{4}}+\mathcal{O}\left(\Delta x^{4}, \beta_{\ell} \Delta x^{3}, \varepsilon \Delta x^{2}, \sigma^{2} \Delta x^{2}\right)
\end{gathered}
$$

where $C r=\sqrt{h} \Delta t / \Delta x$. Therefore, the proposed scheme achieves the same accuracy and stability characteristics for both flat and mild curvature bottoms.

\section{ACKNOWLEDGEMENTS}

The authors would like to thank the two anonymous reviewers for their helpful comments.

\section{REFERENCES}

1. Boussinesq J. Théorie des ondes et des remous qui se propagent le long d'un canal rectangulaire horizontal, en communiquant au liquide contenu dans ce canal des vitesses sensiblement pareilles de la surface au fond. Journal de Mathématiques Pures et Appliqués, Série II 1872; 17:55-108.

2. Serre F. Contribution à l'étude des écoulements permanents et variables dans les canaux. La Houille Blanche 1953; 3:374-388, 830-872.

3. Peregrine DH. Long waves on a beach. Journal of Fluid Mechanics 1967; 27:815-827.

4. Zelt JA. The run-up of nonbreaking and breaking solitary waves. Coastal Engineering 1991; 15:205-246.

5. Schäffer HA, Madsen PA, Deigaard R. A Boussinesq model for waves breaking in shallow water. Coastal Engineering 1993; 20:185-202.

6. Antunes do Carmo JS, Seabra-Santos FJ. On breaking waves and wave-current interaction in shallow water: a 2DH finite element model. International Journal for Numerical Methods in Fluids 1996; 22:429-444.

7. Kennedy AB, Chen Q, Kirby JT, Dalrymple RA. Boussinesq modeling of wave transformation, breaking, and runup. I: 1D. Journal of Waterway, Port, Coastal, and Ocean Engineering 2000; 126:39-47.

8. Chen Q, Kirby JT, Dalrymple RA, Kennedy AB, Chawla A. Boussinesq modeling of wave transformation, breaking, and runup. II: 2D. Journal of Waterway, Port, Coastal, and Ocean Engineering 2000; 126:48-56.

9. Veeramony J, Svendsen IA. The flow in surf-zone waves. Coastal Engineering 2000; 39:93-122.

10. Nwogu O. An alternative form of the Boussinesq equations for modelling the propagation of waves from deep to shallow water. Journal of Waterway, Port, Coastal, and Ocean Engineering 1993; 119:618-638.

11. Wei G, Kirby JT. Time-dependent numerical code for extended Boussinesq equations. Journal of Waterway, Port, Coastal, and Ocean Engineering 1995; 121(5):251-261.

12. Nadaoka K, Beji S, Nakagawa Y. A fully dispersive weakly nonlinear model for water waves. Proceedings of the Royal Society of London, Series A 1997; 453:303-318.

13. Madsen PA, Schäffer HA. Higher-order Boussinesq-type equations for surface gravity waves: derivation and analysis. Philosophical Transactions of the Royal Society of London, Series A 1998; 356:3123-3184.

14. Gobbi MF, Kirby JT. Wave evolution over submerged sills: tests of a high-order Boussinesq model. Coastal Engineering 1999; 37:57-96.

15. Gobbi MF, Kirby JT, Wei G. A fully nonlinear Boussinesq model for surface waves. Part 2. Extension to $O(k h)^{4}$. Journal of Fluid Mechanics 2000; 405:181-210.

16. Madsen PA, Agnon Y. Accuracy and convergence of velocity formulations for water waves in the framework of Boussinesq theory. Journal of Fluid Mechanics 2003; 477:285-319.

17. Madsen PA, Bingham HB, Liu H. A new Boussinesq method for fully nonlinear waves from shallow to deep water. Journal of Fluid Mechanics 2002; 462:1-30.

18. Abbott MB, Petersen HM, Skovgaard O. On the numerical modelling of short waves in shallow water. Journal of Hydraulic Research 1978; 16(3):173-203. 


\section{A PETROV-GALERKIN FEM FOR THE BOUSSINESQ WAVE MODEL}

19. Abbott MB, McCowan AD, Warren IR. Accuracy of short-wave numerical models. Journal of Hydraulic Engineering 1984; 110(10):1287-1301.

20. Antunes do Carmo JS, Seabra-Santos FJ, Almeida AB. Numerical solution of the generalized Serre equations with the MacCormack finite-difference scheme. International Journal for Numerical Methods in Fluids 1993; 16: $725-738$.

21. Fuhrman DR, Bingham HB. Numerical solutions of fully non-linear and highly dispersive Boussinesq equations in two horizontal dimensions. International Journal for Numerical Methods in Fluids 2004; 44:231-255.

22. Fuhrman DR, Bingham HB, Madsen PA, Thomsen PG. Linear and non-linear stability analysis for finite difference discretizations of high-order Boussinesq equations. International Journal for Numerical Methods in Fluids 2004; 45:751-773.

23. Bradford SF, Sanders BF. Finite-volume models for unidirectional, nonlinear, dispersive waves. Journal of Waterway, Port, Coastal, and Ocean Engineering 2002; 128:173-182.

24. Stansby PK. Solitary wave run up and overtopping by a semi-implicit finite-volume shallow-water Boussinesq model. Journal of Hydraulic Research 2003; 41:639-647.

25. Erduran KS, Ilic S, Kutija V. Hybrid finite-volume finite-difference scheme for the solution of Boussinesq equations. International Journal for Numerical Methods in Fluids 2005; 49:1213-1232.

26. Cienfuegos R, Barthélemy E, Bonneton P. A fourth-order compact finite volume scheme for fully nonlinear and weakly dispersive Boussinesq-type equations. Part I: model development and analysis. International Journal for Numerical Methods in Fluids 2006; 51:1217-1253.

27. Cienfuegos R, Barthélemy E, Bonneton P. A fourth-order compact finite volume scheme for fully nonlinear and weakly dispersive Boussinesq-type equations. Part II: boundary conditions and validation. International Journal for Numerical Methods in Fluids 2007; 53:1423-1455.

28. Katopodes ND, Wu CT. Computation of finite-amplitude dispersive waves. Journal of Waterway, Port, Coastal, and Ocean Engineering 1987; 113:327-346.

29. Antunes do Carmo JS, Seabra Santos FJ, Barthélemy E. Surface waves propagation in shallow water: a finite element model. International Journal for Numerical Methods in Fluids 1993; 16:447-459.

30. Kawahara M, Cheng JY. Finite element method for Boussinesq wave analysis. International Journal of Computational Fluid Dynamics 1994; 2:1-17.

31. Ambrosi B. A new finite element scheme for the Boussinesq equations. Mathematical Models and Methods in Applied Sciences 1997; 7:193-209.

32. Li YS, Liu SX, Yu YX, Lai GZ. Numerical modeling of Boussinesq equations by finite element method. Coastal Engineering 1999; 37:97-122.

33. Walkley M, Berzins M. A finite element method for the one-dimensional extended Boussinesq equations. International Journal for Numerical Methods in Fluids 1999; 29:143-157.

34. Walkley M, Berzins M. A finite element method for the two-dimensional extended Boussinesq equations. International Journal for Numerical Methods in Fluids 2002; 39:865-885.

35. Sørensen OR, Schäffer HA, Sørensen LS. Boussinesq-type modelling using an unstructured finite element technique. Coastal Engineering 2004; 50:181-198.

36. Woo SB, Liu PLF. Finite-element model for modified Boussinesq equations. I: model development. Journal of Waterway, Port, Coastal, and Ocean Engineering 2004; 130:1-16.

37. Woo SB, Liu PLF. Finite-element model for modified Boussinesq equations. II: applications to nonlinear harbor oscillations. Journal of Waterway, Port, Coastal, and Ocean Engineering 2004; 130:17-28.

38. Woo SB, Liu PLF. A Petrov-Galerkin finite element model for one-dimensional fully non-linear and weakly dispersive wave propagation. International Journal for Numerical Methods in Fluids 2001; 37:541-575.

39. Langtangen HP, Pedersen G. Computational models for weakly dispersive nonlinear water waves. Computer Methods in Applied Mechanics and Engineering 1998; 160:337-358.

40. Yu CC, Heinrich JC. Petrov-Galerkin methods for the time-dependent convective transport equation. International Journal for Numerical Methods in Engineering 1986; 23(5):883-901.

41. Pedersen G. Energy conservation and physical optics for discrete long wave equations. Wave Motion 2003; 37:81-100.

42. Hirsch C. Numerical Computation of Internal and External Flows. Volume I: Fundamentals of Numerical Discretization. Wiley: New York, 1992.

43. Avilez-Valente P, Seabra-Santos FJ. A Petrov-Galerkin finite element scheme for the regularized long wave equation. Computational Mechanics 2004; 34:256-270.

44. Teng MH, Wu TY. Nonlinear water waves in channels of arbitrary shape. Journal of Fluid Mechanics 1992; 242:211-233. 


\section{P. AVILEZ-VALENTE AND F. J. SEABRA-SANTOS}

45. Teng MH. Solitary wave solution to Boussinesq equations. Journal of Waterway, Port, Coastal, and Ocean Engineering 1997; 123:138-141.

46. Dingemans MW. Comparison of computations with Boussinesq-like models and laboratory measurements. Technical Report Project 1: Waves, MAST-G8M, Delft Hydraulics, Delft, NL, 1994.

47. Yu CC, Heinrich JC. Petrov-Galerkin method for multidimensional, time-dependent, convective-diffusion equations. International Journal for Numerical Methods in Engineering 1987; 24(11):2201-2215. 